\title{
MIRD Pamphlet No. 27: MIRDcell V3, a revised software tool for multicellular dosimetry and bioeffect modeling
}

\author{
Sumudu Katugampola ${ }^{1}$, Jianchao Wang ${ }^{1}$, Alex Rosen, and Roger W. Howell \\ ${ }^{1}$ Sumudu Katugampola and Jianchao Wang are co-first authors
}

Division of Radiation Research, Department of Radiology, New Jersey Medical School, Rutgers University, Newark, NJ 07103, USA

In collaboration with the SNMMI MIRD Committee: Vikram Adhikarla, Rachel Marie Bartlett, Wesley E. Bolch, Yuni K. Dewaraja, William D. Erwin, Darrell R. Fisher, Robert Francois Hobbs, Roger W. Howell, Adam L. Kesner, Richard Laforest, Joseph G. Rajendran, George Sgouros, and Pat B. Zanzonico (Chair)

\section{Corresponding Author and to whom questions and suggestions should be directed:}

Roger W. Howell, Distinguished Professor

Division of Radiation Research, Department of Radiology and Center for Cell Signaling

Rutgers New Jersey Medical School, Cancer Center, F-1208

205 S. Orange Avenue, Newark, NJ 07103, USA

Tel: 973-972-5067, Fax: 973-972-6474

rhowell@rutgers.edu

First authors:

Sumudu Katugampola, PhD is a postdoctoral fellow and

Jianchao Wang, MS is a research assistant.

Word count: 7001

\section{Running title: MIRDcell V3}

Immediate Open Access: Creative Commons Attribution 4.0 International License (CC BY) allows users to share and adapt with attribution, excluding materials credited to previous publications.

License: https://creativecommons.org/licenses/by/4.0/. Details: https://jnm.snmjournals.org/page/permissions.

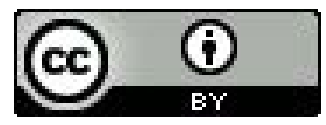




\section{GRAPHICAL ABSTRACT}

\section{MIRDcell V3: Multicellular-Level Dosimetry for RPT}

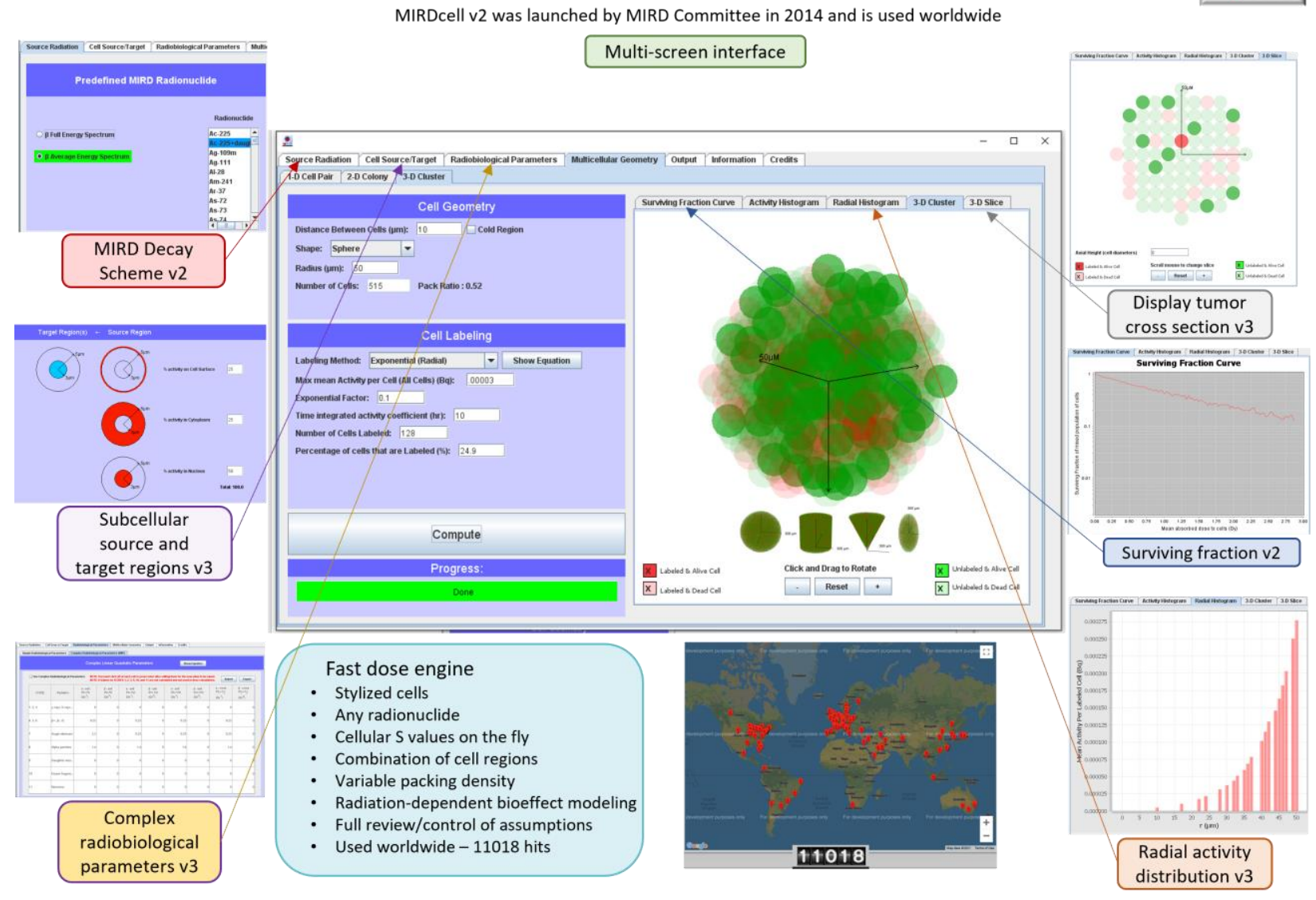




\section{ABSTRACT}

Radiopharmaceutical therapy is growing rapidly. However, yet to be addressed is the implementation of methods to plan treatments for circulating tumor cells, disseminated tumor cells, and micrometastases. Given the capacity of radiopharmaceuticals to specifically target and kill single cells and multicellular clusters, a quality not available in chemotherapy and external beam radiation therapy, it is important to develop dosimetry and bioeffect modeling tools that can inform radiopharmaceutical design and predict their effect on the microscopic disease. This pamphlet describes a new version of MIRDcell, a software tool that was initially released by the Medical Internal Radiation Dose (MIRD) Committee several years ago.

\section{Methods}

MIRDcell V3 uses a combination of analytical and Monte Carlo methods to conduct dosimetry and bioeffect modeling for radiolabeled cells within planar colonies and multicellular clusters. Three worked examples are provided to assist users to learn old and new features of MIRDcell, and test its capacity to recapitulate published responses of tumor cell spheroids to radiopharmaceutical treatments. Prominent capabilities of the new version include radially dependent activity distributions, user-imported activity distributions, cold-regions within the cluster, complex bioeffect modeling that accounts for radiation type and subcellular distribution, and a rich table of output data for subsequent analysis.

\section{Results}

MIRDcell V3 effectively reproduces experimental responses of multicellular spheroids to uniform and nonuniform distributions of therapeutic radiopharmaceuticals.

\section{Conclusion}

MIRDcell is a versatile software tool that can be used for educational purposes and design of radiopharmaceutical therapies. 


\section{Keywords}

Dosimetry

Radionuclide

Multicellular cluster

Cell survival

Nonuniform activity distribution 


\section{INTRODUCTION}

The widespread use of ${ }^{223} \mathrm{Ra}$ dichloride $\left(\mathrm{Xofigo}^{\circledR}\right)$ and ${ }^{177}$ Lu-DOTATATE (LUTATHERA ${ }^{\circledR}$ ) has rejuvenated radiopharmaceutical therapy (RPT) of cancer. RPT delivers radioactive drugs to the primary tumor, metastases, disseminated tumor cells (DTC), and circulating tumor cells (CTC). Different classes of radionuclides are used for therapy including $\alpha-, \beta-$, and Auger electron emitters (1). The different ranges of these radiations in tissue, and their differences in relative biological effectiveness (RBE), contribute to the complexity of predicting therapeutic efficacy and normal tissue toxicity (1). However, like external beam radiation therapy (EBRT), the future of RPT will depend in part on our capacity to plan treatments that maximize therapeutic effect while minimizing adverse effects in normal tissues. Key to the long-term success of RPT is to implement strategies that overcome limitations of the intrinsic nonuniform uptake of radiopharmaceuticals by cancer cells that can impact our capacity to sterilize tumors, metastases, DTC, and CTC.

While treatment of primary tumors, distant metastases visible by external imaging, and microscopic metastases in locoregional lymph nodes can be addressed with external beams of radiation, most micrometastases, DTC, and CTC cannot. There are commercial tools, based on external imaging, to assist with calculating absorbed dose to macroscopic disease in the context of both EBRT and RPT. The resulting absorbed doses have been used to predict response of tumor and normal tissues. However, there is a dearth of readily available tools that can be used to optimize and plan RPT of microscopic disease.

In 2014, the Medical Internal Radiation Dose (MIRD) Committee released MIRDcell V2.0.15, a Java applet, to address the need for software tools for dosimetry and bioeffect modeling of microscopic disease treated with RPT. The software interface and its capabilities were described in MIRD Pamphlet No. 25 (2). That version, and a later version V2.0.16, ran on 
a web browser until 2017 when web browser support for Java applets was discontinued due to security concerns. MIRDcell V2.1, a Java application, was released in 2017 to eliminate the web browser requirement and permit the program to run as an application on the user computer.

This new version of our MIRDcell software application, MIRDcell V3, was created in collaboration with the MIRD Committee. MIRDcell V3 can run on all operating systems supporting Java. The software can model radiation absorbed dose and cell survival responses in single cells, cell pairs, and 2- and 3-dimensional cell populations. Two-dimensional cell populations are constrained to lie on a plane (e.g. monolayer cell cultures), whereas 3dimensional populations can be organized within a variety of geometries. The organization of the tabs and options within each tab, and other important details regarding the version history, are provided in detail in the downloadable User Manual. The nomenclature used is consistent with dosimetric terminology published in MIRD Pamphlet No. 21 (3). The app and User Manual can be downloaded via https://mirdsoft.org or directly at https://mirdcell.njms.rutgers.edu/. The primary purpose of this present MIRD Pamphlet is to describe some of the changes in interactive features, new activity distributions, and new bioeffect models that have been added to the software. More importantly, this pamphlet provides several examples of how to use these new features. 


\section{MATERIALS AND METHODS}

\section{Preamble}

The distribution of radioactivity within small tissue elements can have a profound effect on the absorbed dose distribution and correspondingly, the response of the tissue. Aside from other factors, the absorbed dose distribution and biological response are strongly dependent on the type, yield, and energy of the radiations emitted by the radionuclide and its subcellular distribution. Most notably are radionuclides that decay by electron capture and/or internal conversion (e.g. $\left.{ }^{111} \mathrm{In},{ }^{123} \mathrm{I},{ }^{125} \mathrm{I}\right)$ which are followed by the emission of a shower of low-energy Auger electrons. Auger electrons deposit their energy over subcellular dimensions and therefore these radionuclides invariably produce nonuniform absorbed-dose distributions at all spatial levels $(4,5)$. Similarly, the short range of alpha particles in biological tissues $(40-100 \mu \mathrm{m})$ also leads to nonuniform dose distributions from radionuclides such as ${ }^{223} \mathrm{Ra},{ }^{225} \mathrm{Ac}$, and other alpha particle emitters of potential use for RPT (5-10). Medium- and high-energy beta particle emitters such as ${ }^{177} \mathrm{Lu}$ and ${ }^{90} \mathrm{Y}$ have a greater degree of cross-irradiation because their mean range in tissue is at least several hundred microns. However, the nonuniform distribution of these radionuclides invariably leads to nonuniform dose distributions as well (11-15).

While the distributions of absorbed dose that arise from nonuniform distributions of radioactivity are important, an additional factor that determines biological response is whether a given absorbed dose arises from radioactive decays within a cell itself (self-dose) or from decays in surrounding cells or other parts of the body (cross-dose). The response of a cell to self-dose from a radiopharmaceutical can be different than its response to cross-dose from the same radiopharmaceutical. This is most notable for Auger electron emitters where the relative biological effectiveness (RBE) for the self-dose can be an order of magnitude greater than the 
RBE for cross-dose (16). This has also been observed for DNA-incorporated beta-particle emitters, where the self-dose from ${ }^{131}$ I was three times more lethal than the cross-dose (17).

There is a growing body of experimental data on the biological effects of nonuniform distributions of radioactivity at the multicellular level (17-23). These findings can have significant consequences for therapeutic uses of these and other radionuclides. MIRDcell V3 provides new tools that can be used to assist in understanding the dependence of radiopharmaceutical efficacy on numerous factors such as: i) radiation type and energy, ii) distribution at the subcellular, cellular, and multicellular levels, and iii) spatial arrangement of the cells within the multicellular structure (two-dimensional plane (e.g. colony), three-dimensional cluster, and packing density). These capabilities and new bioeffect modeling features are expanded upon through examples below, can be helpful in designing RPT strategies.

\section{Source Radiation tab}

This tab allows the user to select the radioactivity in the source cells (i.e. cells labeled with radioactivity). Three choices are available: (1) predefined MIRD radionuclide, (2) monoenergetic particle emitter, and (3) user-defined radionuclide. User defined radionuclides include decay chains for ${ }^{211} \mathrm{At},{ }^{213} \mathrm{Bi},{ }^{223} \mathrm{Ra}$, and ${ }^{225} \mathrm{Ac}$. Details regarding the differences between these options can be found in the User Manual.

\section{Cell Source/Target tab}

As described in detail in Goddu et al. (24) and the MIRD Cellular S Values monograph (25), cells are modeled as two concentric spheres with radii corresponding to those for the nucleus $\left(R_{N}\right)$ and cell $\left(R_{C}\right)$, respectively. The cells are modeled as liquid water of unit density. The eligible source regions are cell (C), cell nucleus (N), cytoplasm (Cy), and cell surface (CS). MIRDcell V3 newly permits the user to distribute the activity between $\mathrm{N}, \mathrm{Cy}$, and CS. The 
eligible target regions for which the radiation absorbed dose is calculated, and used for bioeffect modeling, are $\mathrm{C}, \mathrm{N}$, and Cy. The addition of cytoplasm as a target is new to MIRDcell V3. No limit has been set on the maximum cell radius, however, extensive testing has only been conducted up to $10 \mu \mathrm{m}$. Although the algorithms should be adequate for calculating absorbed doses to larger spheres, caution should be exercised when interpreting results for $R_{C}>10 \mu m$. To facilitate this option, MIRDcell V3 now allows entering the radii in the text box. Users should be mindful that photons are ignored in this and earlier versions of MIRDcell; photon contributions to the absorbed dose can become significant for large sphere sizes.

\section{Radiobiological Parameters tab}

MIRDcell enables the user to model the surviving fraction (SF) of cells in a specified cell population based on the calculated absorbed doses to the individual cells. Two options are available in V3 for calculating the probability that a given cell survives:

a) Simple Radiobiological Parameters. As in MIRDcell V2.1 (2), a modified linearquadratic (LQ) model is used to calculate the probability $P\left(n_{\mathrm{k}}\right)$ that a given cell, $n_{\mathrm{k}}$, survives $(26,27)$,

$$
P\left(n_{k}\right)=e^{-\alpha_{\text {self }} D_{\text {self }}-\beta_{\text {self }} D_{\text {self }}^{2}} \times \mathrm{e}^{-\alpha_{\text {cross }} D_{\text {cross }}-\beta_{\text {cross }} D_{\text {cross }}^{2}},
$$

where $\alpha_{\text {self }}$ and $\beta_{\text {self }}$ characterize the response of the cell to self-dose $\left(D_{\text {self }}\right), \alpha_{\text {cross }}$ and $\beta_{\text {cross }}$ characterize the cellular response to cross-dose ( $\left.D_{\text {cross }}\right)$, and the effect of self- and cross-dose are independent $(5,17,28)$. The distinction between self- and cross-dose is often required for Auger electron emitters $(18,29)$, and sometimes for beta-particle emitters when they are DNAincorporated (17). The determination of whether a given cell survives (alive) or not (dead) is determined by a Monte Carlo method where the surviving probability, calculated as described above, is compared with a randomly generated number. 
b) Complex Radiobiological Parameters (new). A new feature of V3 is the capacity to specify LQ parameters not only for self-dose and cross-dose, but also independently for each type of radiation (alpha, beta, Auger, etc.) and for each target region (C, N, Cy). A modified LQ model is again implemented. For example, when the cell nucleus, $\mathrm{N}$, is the target region and the source radiation type is designated by ICODE, the probability that the $k^{\text {th }}$ cell survives the insult is given by Eq.2.

$$
\begin{aligned}
& P_{I C O D E}\left(N_{k}\right)=e^{-\alpha_{I C O D E}^{\text {self }}\left(N_{k} \leftarrow N_{k}\right) D_{I C O D E}^{\text {self }}\left(N_{k} \leftarrow N_{k}\right)-\beta_{I C O D E}^{\text {self }}\left(N_{k} \leftarrow N_{k}\right)\left[D_{I C O D E}^{\text {self }}\left(N_{k} \leftarrow N_{k}\right)\right]^{2}} \\
& \times e^{-\alpha_{I C O D E}^{\text {self }}\left(N_{k} \leftarrow C y_{k}\right) D_{I C O D E}^{\text {self }}\left(N_{k} \leftarrow C y_{k}\right)-\beta_{I C O D E}^{\text {self }}\left(N_{k} \leftarrow C y_{k}\right)\left[D_{I C O D E}^{\text {self }}\left(N_{k} \leftarrow C y_{k}\right)\right]^{2}} \\
& \times e^{-\alpha_{I C O D E}^{\text {self }}\left(N_{k} \leftarrow C S_{k}\right) D_{I C O D E}^{\text {self }}\left(N_{k} \leftarrow C S_{k}\right)-\beta_{I C O D E}^{\text {self }}\left(N_{k} \leftarrow C S_{k}\right)\left[D_{I C O D E}^{\text {self }}\left(N_{k} \leftarrow C S_{k}\right)\right]^{2}} \\
& \times e^{-\alpha_{I C O D E}^{\text {cross }}\left(N_{k} \leftarrow N_{j}\right) D_{I C O D E}^{\text {cross }}\left(N_{k} \leftarrow N_{\text {numcell }}\right)-\beta_{I C O D E}^{\text {cross }}\left(N_{k} \leftarrow N_{j}\right)\left[D_{I C O D E}^{\text {cross }}\left(N_{k} \leftarrow N_{\text {numcell }}\right)\right]^{2}} \\
& \times e^{-\alpha_{I C O D E}^{\text {cross }}\left(N_{k} \leftarrow C y_{j}\right) D_{I C O D E}^{\text {cross }}\left(N_{k} \leftarrow C y_{\text {numcell }}\right)-\beta_{I C O D E}^{\text {cross }}\left(N_{k} \leftarrow C y_{j}\right)\left[D_{I C O D E}^{\text {cross }}\left(N_{k} \leftarrow C y_{\text {numcell }}\right)\right]^{2}} \\
& \times e^{-\alpha_{I C O D E}^{\text {cross }}\left(N_{k} \leftarrow C S_{j}\right) D_{I C O D E}^{\text {cross }}\left(N_{k} \leftarrow C S_{\text {numcell }}\right)-\beta_{I C O D E}^{\text {cross }}\left(N_{k} \leftarrow C S_{j}\right)\left[D_{I C O D E}^{\text {cross }}\left(N_{k} \leftarrow C S_{\text {numcell }}\right)\right]^{2}}
\end{aligned}
$$

where,

$$
D_{\text {ICODE }}^{\text {self }}\left(N_{k} \leftarrow N_{k}\right)=f_{N} \widetilde{A}\left(C_{k}\right) S_{I C O D E}^{\text {self }}\left(N_{k} \leftarrow N_{k}\right)
$$

The ICODEs for the different radiation types are as defined in the MIRD Radionuclide Decay Scheme monograph (30). Here, $f_{N}$ is the fraction of cell activity in the nucleus, $\tilde{A}\left(C_{k}\right)$ is the timeintegrated activity in the source region $N_{k}$ and $S_{I C O D E}^{\text {self }}\left(N_{k} \leftarrow N_{k}\right)$ is the self S-value corresponding to the absorbed dose per decay from $N_{k} \leftarrow N_{k}$ and is given by Eq. 4:

$$
S_{I C O D E}^{\text {self }}\left(N_{k} \leftarrow N_{k}\right)=\sum_{\text {irad }=1}^{\text {iradN }} \frac{\Delta_{I C O D E, \text { irad }} \phi_{I C O D E, \text { irad }}\left(N_{k} \leftarrow N_{k}\right)}{m\left(N_{k}\right)} .
$$

Where the sum runs through all irad $N$ radiations of type ICODE, $\Delta_{I C O D E \text {,irad }}$ is the mean energy emitted per nuclear transition of the irad $^{\text {th }}$ radiation of type ICODE, $\phi_{I C O D E \text {, irad }}\left(N_{k} \leftarrow N_{k}\right)$ is the fraction of energy emitted from the source region $N_{k}$ that is absorbed in the target region $N_{k}$ of 
the $\mathrm{irad}^{\text {th }}$ radiation of type ICODE. The terms corresponding to the self-dose from other cell compartments of the same cell (Cy, CS) can be written similarly, as can the terms corresponding to the cross-doses from other cells. Finally, the overall probability of the $k^{\text {th }}$ cell surviving, after the effects of all radiation types on the $k^{\text {th }}$ cell nucleus $N_{k}$, is written as:

$$
P\left(N_{k}\right)=\prod_{I C O D E=1}^{\text {Number of ICODES }} P_{I C O D E}\left(N_{k}\right)
$$

Here, MIRDcell adopts an independent interaction model where the effect of each radiation type is considered independent of the other. As in prior versions of MIRDcell, the determination of whether a given cell survives (alive) or not (dead) is determined by a Monte Carlo method where the probability of survival, calculated as described above, is compared with a random number $(0 \leq x \leq 1)$. See User Manual for details.

A complete set of equations for all possible scenarios of source/target regions is provided in the User Manual. Default values are arbitrary and the user is cautioned to enter values that are relevant to their application. The user is provided with the option of importing a desired set of LQ parameters and also saving a set of custom parameters used in the model.

\section{Multicellular Geometry tab}

\section{Cluster Geometry}

As in MIRDcell V2.1, MIRDcell V3 has three basic geometric configurations of spherical cells: 1-dimensional (1-D), 2-dimensional (2-D), and 3-dimensional (3-D). These are summarized below and details are provided in the User Manual.

The 1-D option is presented in the 1-D cell Pair tab and it is used to calculate the selfand cross-doses for a pair of cells. The user can set the distance (d) between the centers of two cells. The self-dose and cross-dose $S$ coefficients (formerly $S$ values) are calculated using 
analytical methods based on range-energy relationships for electrons $(31,32)$ and alpha particles (33) as described in the Supplemental Data.

The 2-D option is used to create a cell population that resides on a plane (i.e. colony). The cell packing density can be specified by changing the distance between the cells, and the shape (circle, rectangle, ellipse) and dimensions of the colony can be set.

The 3-D option is attained by extending the planar cell configuration to a threedimensional cluster. The shape of the cluster is selectable as a sphere, ellipsoid, rod, or cone Cell packing density and dimensions of the cluster are specified by the user. The cluster is assembled in a 3-dimensional Cartesian coordinate system in a close-packed cubic lattice.

\section{Cell Labeling}

Random Activity Distributions. The distribution of activity among the labeled cell population is set by the user in the 2-D Cluster and 3-D Cluster configurations. As in MIRDcell V2.1, both the 2-D and 3-D configurations offer several random distributions where the activity is distributed among the labeled cells according to a uniform, normal, or log-normal distribution. Labeled cells are selected randomly and each cell randomly assigned an initial activity according to user-selected distribution. A uniform activity distribution among the labeled cells implies that each labeled cell has the same initial activity $A$ in its source region. In the normal distribution, the initial activity per cell is distributed according to the probability density function:

$$
f(A)=\frac{1}{A \sigma \sqrt{2 \pi}} e^{\frac{-(A-\langle A\rangle)^{2}}{2 \sigma^{2}}}
$$

where $\langle A>$ is the mean initial activity per cell and $\sigma$ is the standard deviation of the mean. In the case of the lognormal distribution, the activity per cell is distributed according to the probability density function: 


$$
f(A)=\frac{1}{A \sigma \sqrt{2 \pi}} e^{\frac{-\left(\ln A-\left(\ln \langle A\rangle-\sigma^{2} / 2\right)\right)^{2}}{2 \sigma^{2}}}, \quad A>0
$$

where $\sigma$ is the lognormal shape-parameter. The functional form of the three distributions mentioned above are best viewed in the "Activity Histogram" tab.

Radial Activity Distributions (new). New functionality in MIRDcell V3 includes both built-in and user-provided radial activity distributions. The built radial distributions are linear, exponential, polynomial and four-parameter log-normal distributions. Polynomial distributions up to the $10^{\text {th }}$ degree are possible by setting parameters accordingly. In all the radial activity distributions, $r=0$ corresponds to the center of the cell cluster. It is important to note that the user-defined activity distribution feature is only available for the spherical cluster geometry. Furthermore, for the ellipsoid cluster geometry, only the standard normal, log-normal and uniform activity distributions are available as cell labeling methods. Details regarding each distribution are provided in the User Manual.

Drug Penetration Depth (new). MIRDcell V2 assumed that the radiopharmaceutical penetrates all the way into the cell cluster. MIRDcell V3 now provides the option of creating a cold region at the center of the cluster and specifying the depth (in $\mu \mathrm{m}$ ) to which the drug penetrates into the cluster from its outer surface. This situation is common for cluster with radii > $50 \mu \mathrm{m}$. The cold region at the center of the cluster will contain unlabeled cells. The various activity distributions described in the previous paragraph can be assigned to the outer region of the cluster that has the labeled cells. The complex algebraic algorithms that are used to label cells according to the drug penetration depth are provided for different geometries in the User Manual. 


\section{Visualization of radial distributions (new)}

MIRDcell V3 now has tools to visualize the radial distributions of mean activity per labelled cell, mean self-dose to labeled cells, mean cross-dose to labeled cells, mean crossdose to unlabeled cells, mean decays per labeled cell, and the mean dose to all cells. This feature can be accessed from the "Radial Histogram" tab which is only available for 3-D cluster geometries. It is an important tool for checking that the specified activity distribution meets the user's expectations.

\section{Visualization of tomographic sections (new)}

Visual representations of the 3-D cell cluster with color-coded labeled/unlabeled and alive/dead cells is accessible from the "3D Cluster" tab. New to MIRDcell V3 is views of tomographic sections (illustrated in the worked example below) of the 3-D geometry in the "3D Slice" tab. The tomographic sections of each layer of cells (specified in cell diameters) can be viewed by scrolling the mouse (Figure 1). 


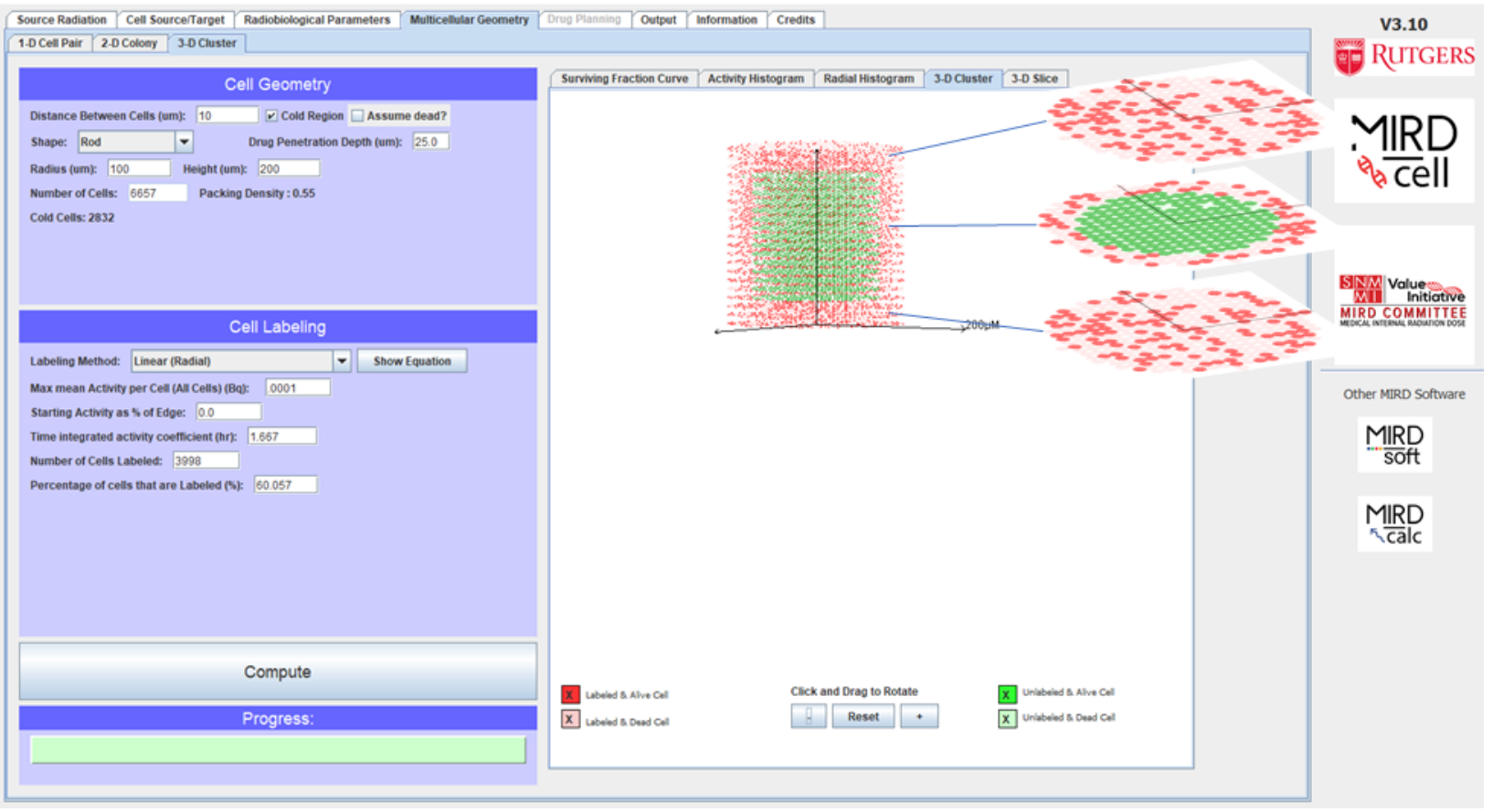

Figure 1. View of three tomographic sections of a rod-shaped cluster of cells with a cold region in the interior. Red cells are labeled with a radionuclide. Green cells are unlabeled. Opaque cells are alive and translucent cells are dead. Blue lines point to the tomographic section of the corresponding cell layer.

\section{Surviving fraction and tumor control probability (new)}

In MIRDcell V2.1, the SF of a cell cluster can be plotted as a function of mean activity per cell $(\mathrm{Bq})$, mean absorbed dose to cells (Gy), mean activity per labeled cell $(\mathrm{Bq})$, mean absorbed dose to labeled cells (Gy), and mean absorbed dose to unlabeled cells (Gy). New to MIRDcell V3 is mean decays per cell and mean decays per labeled cell. Also new is presentation of the tumor control probability (TCP) on the vertical axis which can be visualized as a function of any of the domains specified above. The TCP is calculated using two different approaches. In the first approach, the TCP is calculated using the Poisson Model expression (34): 


$$
T C P(D)=(1-S F(D))^{n}
$$

where $S F(D)$ is the surviving fraction at a mean absorbed dose $D$ and $n$ is the number of cells in the cluster. The Poisson model of TCP works under the assumption that the number of surviving cells are Poisson distributed with an average $n S F(D)$. The second approach takes the survival probability of each cell into account when calculating the $\operatorname{TCP}(22,35)$. The TCP is calculated using the following expression.

$$
T C P=\prod_{i=1}^{n}\left(1-P_{i}\right)
$$

Here, $P_{i}$ is the survival probability of the $i^{\text {th }}$ cell.

\section{Output (new)}

Similar to MIRDcell V2.1, in MIRDcell V3, the output data are written to two boxes in the Output tab. The right-hand box of the Output contains the cellular self- and cross-dose $S$ coefficients for all target $\leftarrow$ source combinations. The left-hand box of the Output contains most of the information and data used to calculate the absorbed doses and bioeffect. These data are used to create the various plots that are available in "Multicellular Geometry" tab. New information in the left-hand box of MIRDcell V3 includes additional input information and the option of saving the Output data as a .txt file. More granular data is provided as well including absorbed doses from each radiation type, radial dose distributions, and other important data used to make the plots. 


\section{WORKED EXAMPLES}

In this section, the overall functionality and accuracy of MIRDcell V3 in predicting biologic response to radiopharmaceuticals is illustrated below with a worked example based on data in the literature.

\section{1. ${ }^{213} \mathrm{Bi}$ bound to cells on the surface of spherical cell clusters}

Data published by Kennel et al. (21) are used in this example to model the radiotoxicity of ${ }^{213} \mathrm{Bi}$ bound to the surface of EMT-6 or Line 1 tumor cells grown as spheroids. Briefly, monoclonal antibody $13 \mathrm{~A}$ to murine CD44 was labeled with ${ }^{213} \mathrm{Bi}\left({ }^{213} \mathrm{Bi}-\mathrm{MAb} 13 \mathrm{~A}\right)$. Only the outer cell layer of the spheroid was labeled such that the activity was localized to a $10 \mu \mathrm{m}$ layer from the spheroid surface. The dosimetry was performed using Monte Carlo methods using an assumed nuclear radius of $5.35 \mu \mathrm{m}$. The average spheroid diameter in their Figure 6 was $250 \mu \mathrm{m}$. Based on their Table 4, we estimated that a cluster of this diameter had 3743 cells. These and other parameters set below were used for both EMT-6 and Line 1 tumor cells as per Kennel et al. (21).

\section{Methods}

a. From the Source Radiation tab in MIRDcell, the $\beta$ Average Energy Spectrum of ${ }^{213} \mathrm{Bi}+$ daughters is selected (Figure 2). By selecting ${ }^{213} \mathrm{Bi}+$ daughters, all radiation types emitted by the daughters of ${ }^{213} \mathrm{Bi}$ decay chain are taken into account in the model and the daughters are assumed to be in equilibrium with the parent. The radiation data are displayed in the Input Data for Calculation box. 


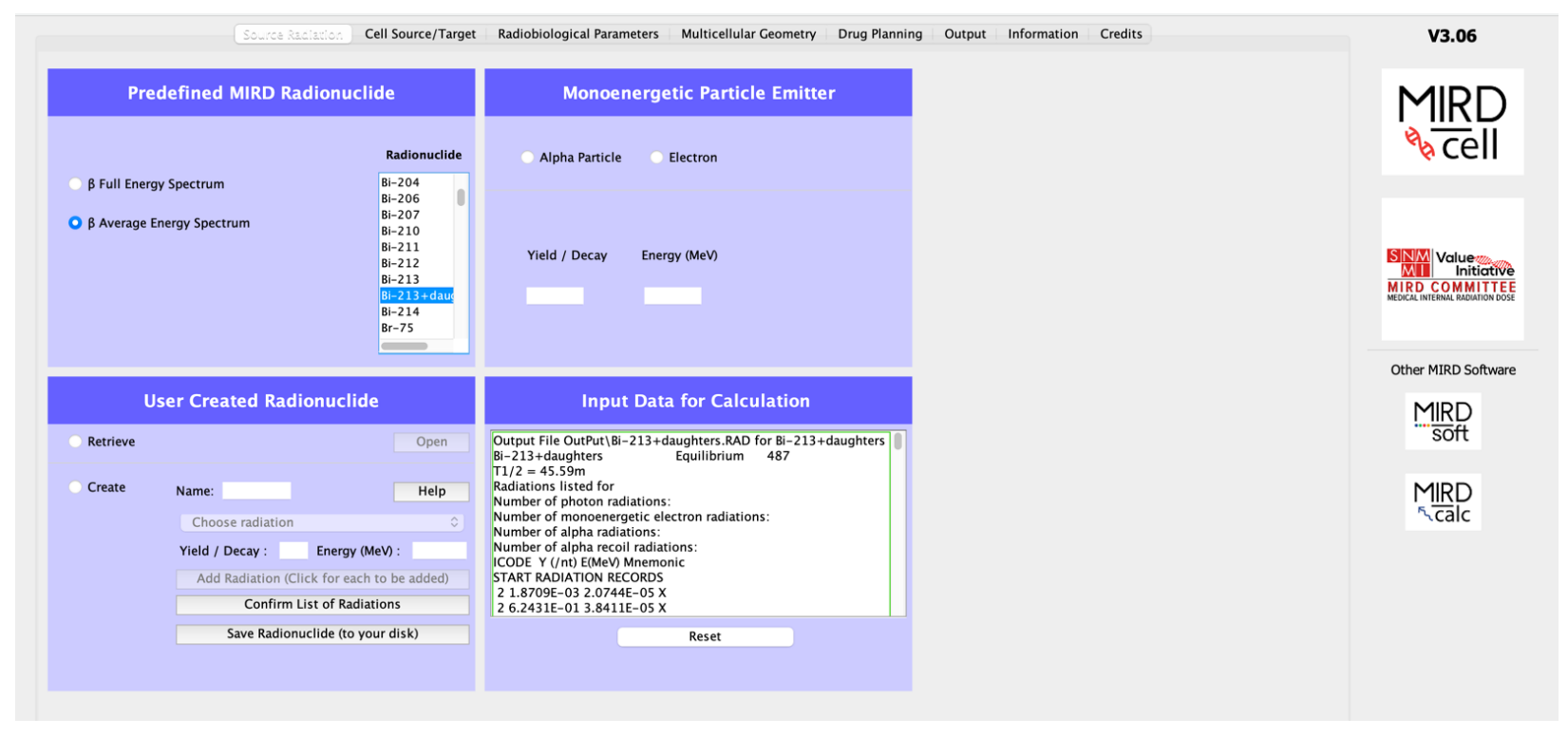

Figure 2: "Source Radiation" Tab

a. In the Cell Source/Target tab the nucleus is selected as the target region and a single source region is the cell surface. The radius of the nucleus is set to $5 \mu \mathrm{m}$. The radius of the cell and the Distance Between Cells (um) in the Multicellular Geometry tab are adjusted until the number of cells in the spherical cluster matches the experimental observations (3473). This requires a cell radius of $6 \mu \mathrm{m}$ and a distance between equal to $13 \mu \mathrm{m}$ (Figure 3).

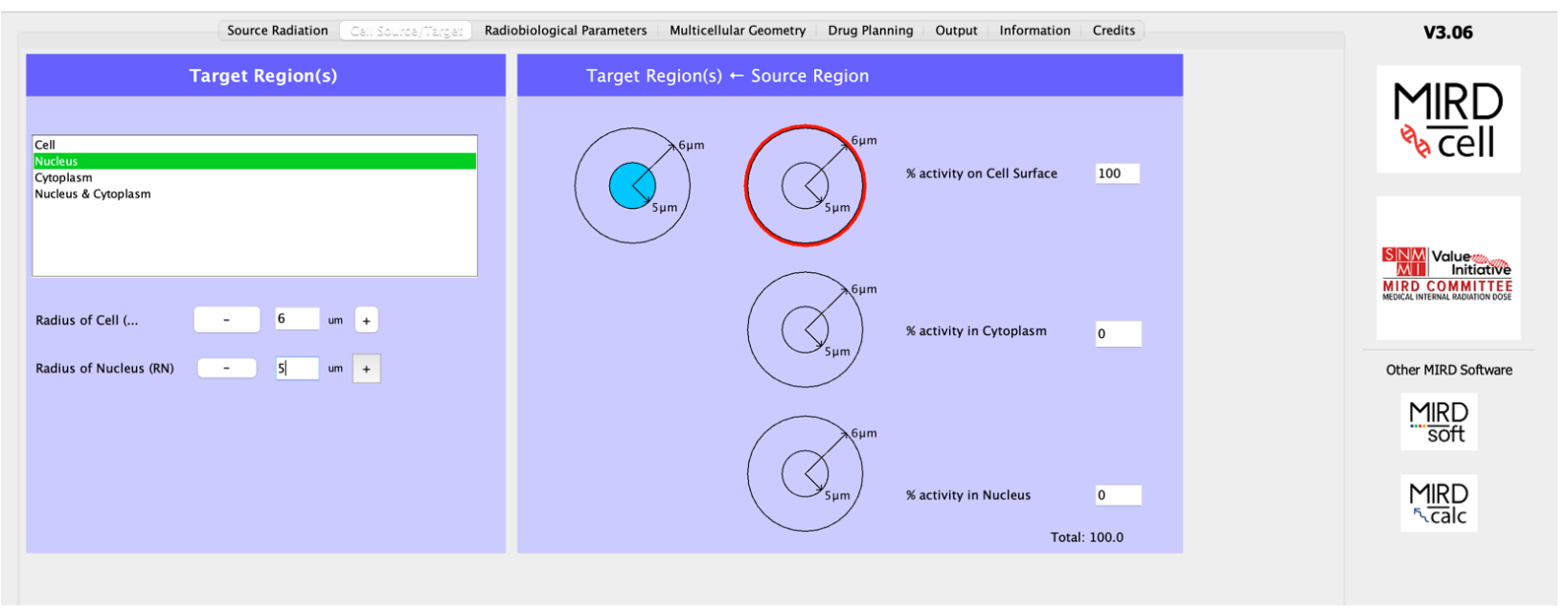

Figure 3: "Cell Source/Target" Tab. 
b. Since the ${ }^{213} \mathrm{Bi}$ decay chain involves many different radiation types and the LQ parameters vary depending on the type of radiation and the target $\leftarrow$ source regions, the Complex Radiobiological Parameters are used rather than the Simple Radiobiological Parameters (Figure 4). Kennel et al. (21) reported a $D_{0}$ of $\sim 1.8$ Gy using a planar alpha particle source for both cell lines used in their experiment. Therefore, the $\alpha$ parameter for alpha particles in the LQ model is changed to $1 / 1.8 \mathrm{~Gy}^{-1} \sim 0.56 \mathrm{~Gy}^{-1}$. Default values are kept for the other radiation types. It is important to note that the model was also run with zeros for all the parameters of Auger electrons and beta particles and the results were the same as were with default values (i.e. Auger electrons and beta particles play no significant role in the response).

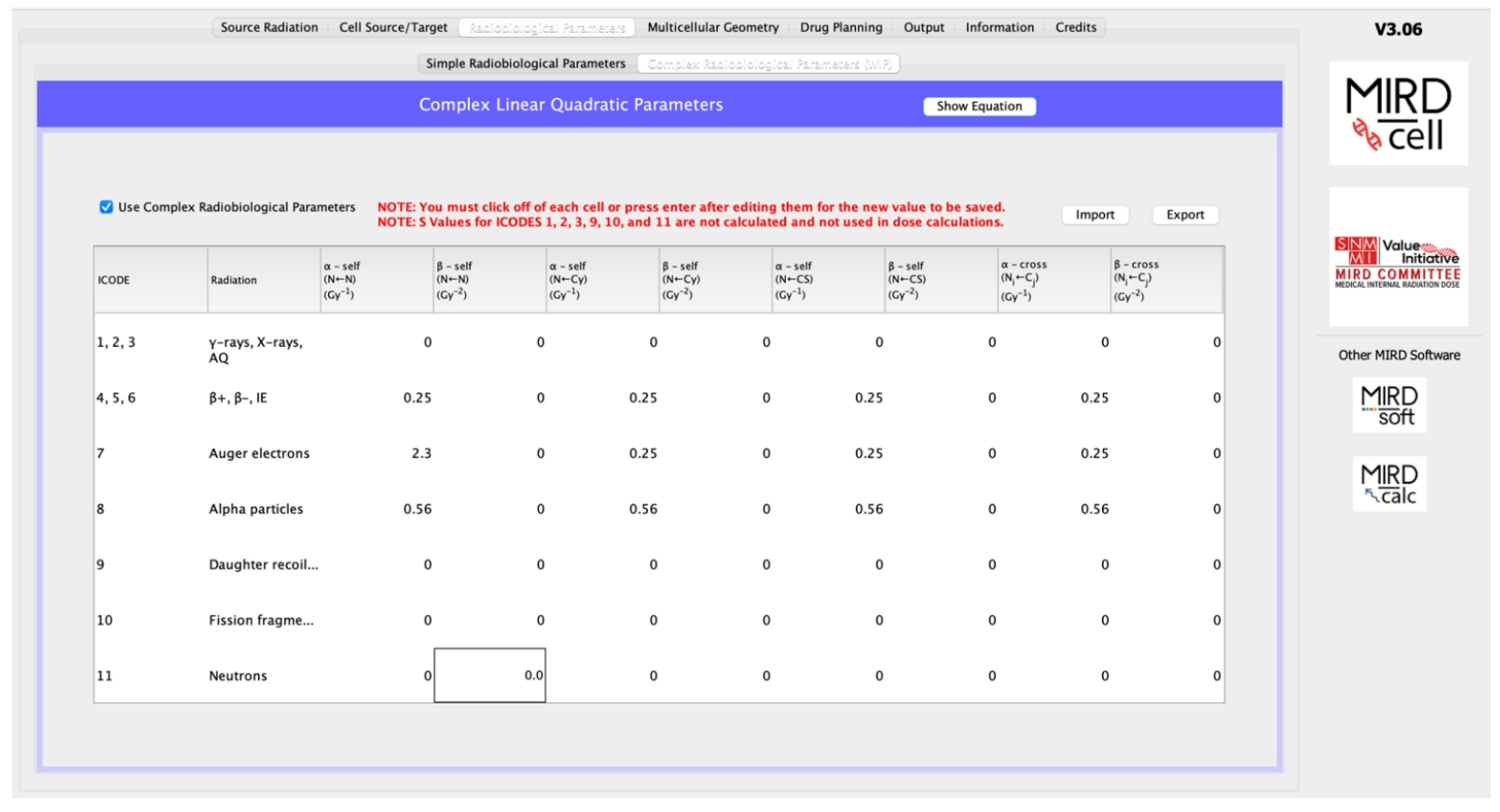

Figure 4:Complex Radiobiological Parameters

c. From the Multicellular Geometry tab, 3-D Cluster is selected and the radius of cluster is set to $125 \mu \mathrm{m}$. The Distance Between Cells (um) is adjusted until the number of cells match the experimental observations (see "b" above). A Drug Penetration Depth (um) of $12 \mu \mathrm{m}$ is set 
and a radial exponential activity distribution is selected from the drop-down menu under Labeling Method; the Exponential Factor was set to 0.4. It important to note that, since the drug penetrates only to a single cell layer $(\sim 12 \mu \mathrm{m})$, the selection of the activity distribution has minimal effect on the rest of the cluster. The Time integrated activity coefficient (hr) is set to $T_{p} / \ln (2)=1.11 \mathrm{~h}$ (where $T_{p}$ is the physical half-life of the radionuclide). Even though what ultimately matters is the product of the time integrated activity coefficient and the maximum mean activity per cell, which gives you the mean number of decays per cell (after correcting $\mathrm{h}$ to $\mathrm{s}$ ), it is helpful to know the time-integrated activity coefficient for reasonability checks. The Percentage of cells that are Labeled (\%) in MIRDcell is set $100 \%$. The Max mean Activity per Cell (All Cells) $(\mathrm{Bq})$ is adjusted until the maximum mean absorbed dose to cells in the MIRDcell Surviving Fraction Curve matched the maximum "Average Dose (Gy)" given in Figure 6 of Kennel et al. (21). When the Compute button is clicked the first time, an error message will pop up indicating that $100 \%$ of the cells cannot be labeled as it exceeds the number of cells within the drug penetration depth; it will automatically set the percentage of labeled cells to the maximum number allowed when the error message is accepted. Therefore, a good rule of thumb is to let the program decide the percentage of labeled cells when a drug penetration depth is specified. Alternatively, if there is a specific desired percentage, set the value prior to clicking the Compute button. Once the model is run, the SF as a function of different domains can be visualized under the Surviving Fraction Curve tab. The Max mean Activity per Cell (All Cells) $(\mathrm{Bq})$ that matched the desired absorbed dose was $0.02 \mathrm{~Bq}$ (Figure 5). 


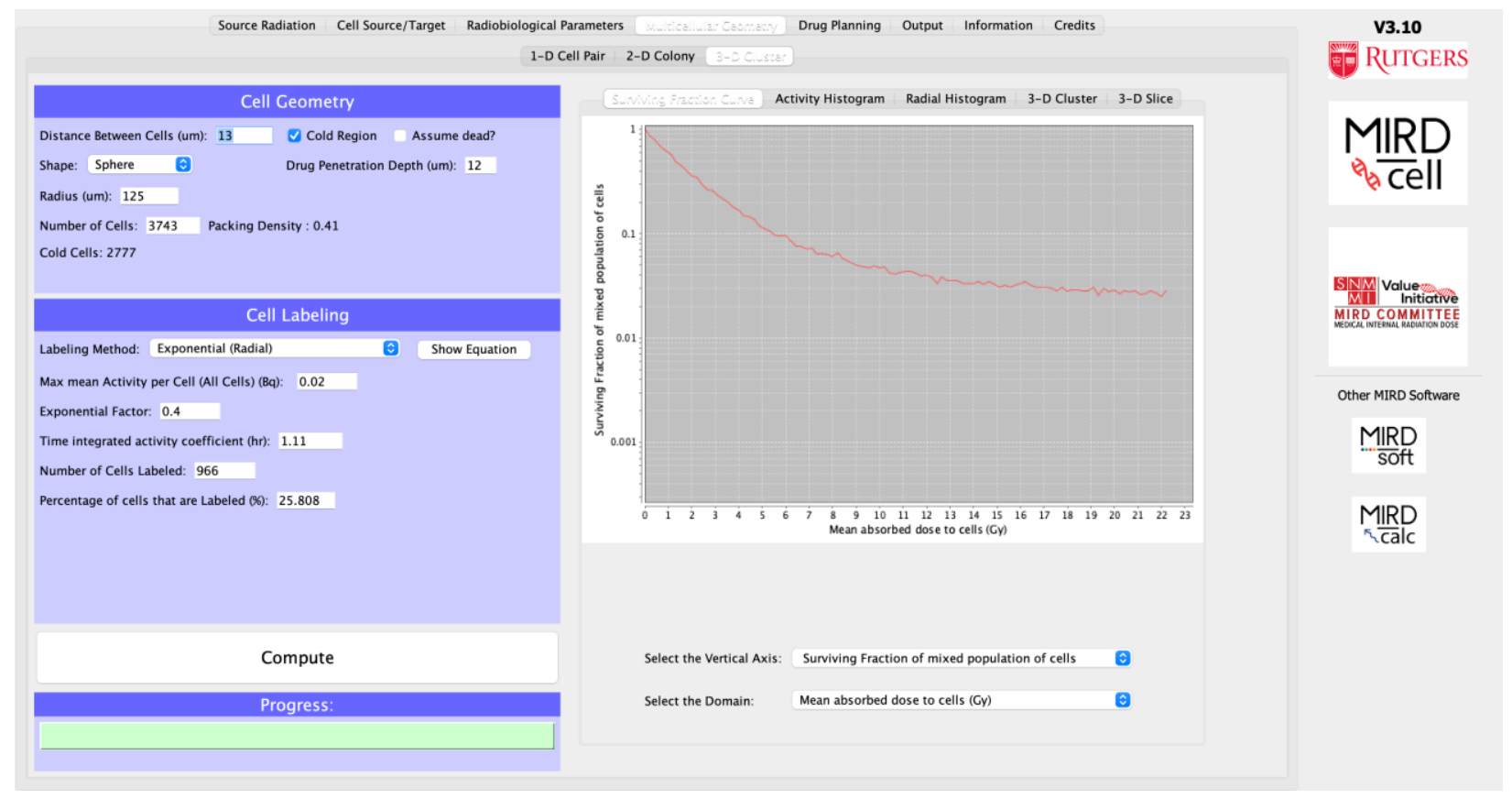

Figure 5: "Multicellular Geometry" Tab. The surviving fraction variation as a function of mean activity per cell is shown on the right.

The radial activity histograms and tomographic sections (Figure 6) of the selected cell cluster geometry are displayed in the Radial Histogram and 3-D Slice tabs, respectively. Tomographic sections of each cell layer can be displayed by specifying the value, in terms of cell diameters, in the box labeled Axial Height. Alternatively, the sections can be scrolled through using the wheel of a mouse. 


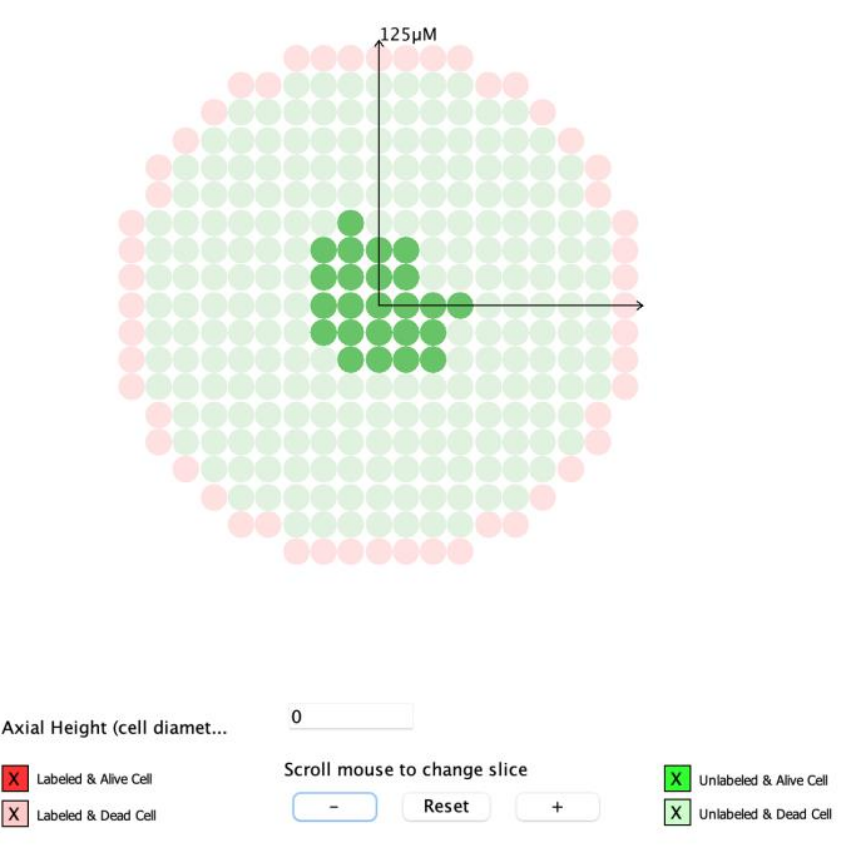

Figure 6: Tomographic section through the center of the spherical cell cluster illustrating the drug penetration depth, labeled cells (red), unlabeled cells (green), alive cells (opaque), and dead cells (translucent). Only unlabeled cells at the center of the cluster are alive.

d. The Output tab lists the values of all the parameters used in the model along with the results (not shown). The left panel lists all the output data used for the plots that be viewed under the Multicellular Geometry tab. The right panel lists all the self-S coefficients and the cross-S coefficients as a function of the distance between the center of the source cell and center of the target cell.

\section{Results and Comparison with Experimental Observations}

Figure 7 illustrates a comparison between the experimental observations for the SF as a function of mean absorbed dose for the two cell lines as taken from Figure 6 of Kennel et al. (21). The reader should focus on the triangles which represent the radiolabeled antibody data. 
The predictions from MIRDcell are given by the red lines. It can be seen from both plots that the data (triangles) are better represented by the MIRDcell prediction than the single component exponential fit used by Kennel et al. (21). Notably, the MIRDcell prediction for the Line 1 cells is superior to that for the EMT-6 cells. Of greatest importance to radiopharmaceutical bioeffect modeling is that MIRDcell predicts the appearance of a tail in the curve as the absorbed dose is increased.
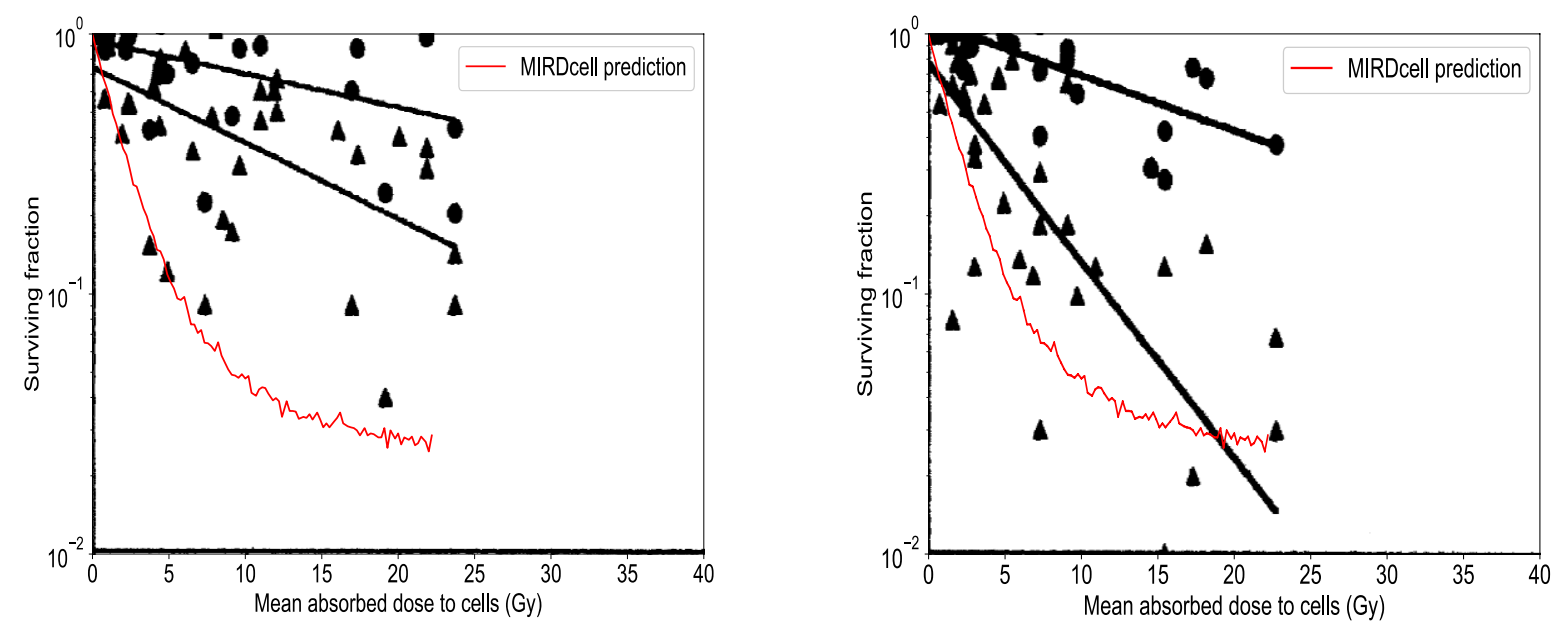

Figure 7: Comparison of MIRDcell prediction with the experimental observations. Original plots extracted from Kennel et al. (21) have been overlayed with the MIRDcell predictions (red). A: EMT-6 cells, B: Line 1 cells. Triangles are data obtained for MAb13A and circles are those obtained for MAb14 which is non-binding with tissue. Solid lines are least squares fits to an exponential function provided by Kennel et al. (21). The MIRDcell simulation was run for MAb13A cells.

Two additional examples are in the Supplemental Data. Example 2 predicts the radiotoxicity of ${ }^{111}$ In-EGF distributed in spherical cell clusters (Supplemental Figs. 1-7). 
Example 3 describes the use of several new features in MIRDcell V3 (Supplemental Figs. 8-10). Additional examples, published previously (2), are in the User Manual.

Also included in the Supplemental Data are comparisons between $\mathrm{S}$ coefficients calculated for 50-keV electrons with MIRDcell and the Monte Carlo code, TOPAS-nBio (36) (Supplemental Figs. 11-12). These S values were used in MIRDcell to generate and compare SF and TCP curves for a 100- $\mu \mathrm{m}$ radius multicellular cluster with uniform or exponential activity distributions (Supplemental Figs. 13-20). S values were calculated similarly for ${ }^{177} \mathrm{Lu}$ and compared (Supplemental Fig. 21). A final comparison for electrons was made with S coefficients that were calculated based on the Emfietzoglou range-energy relationship (Supplemental Fig. 22) (37). Lastly, comparisons between $\mathrm{S}$ coefficients calculated for $5-\mathrm{MeV}$ alpha particles and ${ }^{210} \mathrm{Po}$ are made between MIRDcell and TOPAS-nBio (Supplemental Figs. 23-24).

\section{Discussion}

A number of other codes for multicellular dosimetry and bioeffect modeling have been published over the years $(5,38-40)$. Charlton published a program for multicellular dosimetry that used analytical approaches to predict cell survival in micrometastases consisting of two cell types (41). Hobbs et al. created a GEANT4-based program for multicellular dosimetry with features to calculate tumor control probability (35). Howell et al. expanded on his earlier work cited above by studying the impact of lognormal distributions of activity among the cell population in multicellular clusters (42). Marcatili et al. developed general-purpose software tools to generate randomized $3 \mathrm{D}$ cell culture geometries based on experimentally determined parameters (cell size, cell density, cluster density, average cluster size, cell cumulated activity). Their models were used in conjunction with analytical and Monte Carlo dosimetry calculations to predict the fraction of surviving cells following uptake of ${ }^{177} \mathrm{Lu}$ radiopharmaceuticals (43). Cai et 
al. developed a multicellular model that used MCNP radiation transport (44). Sizeable differences up to about $30 \%$ in the cross-dose $S$ coefficients produced by their code versus MIRDcell V2 were noted. These differences, and their modest impact on SF and TCP, are discussed in the Supplemental Data. The most detailed model was published recently by Raghavan et al. (45). The Raghavan model accounts for time-dependent advection and diffusion of radiopharmaceuticals into cells surrounding the cavity that remains after resecting brain tumors. While MIRDcell does not have similar capability, we are developing a Python code that processes 3-D activity distribution snapshots over time and calculates the radiallydependent time-integrated-activity on a cell-by-cell basis.

Although many multicellular dosimetry programs have been developed, they are largely in the hands of their creators and not available widely for general use. Supplemental Table 1 compares the features of MIRDcell with two codes that are available for users, namely COOLER (46) and PARaDIM (47). While the latter two can accommodate more diverse geometric shapes for the cells, they have a limited scope of other options compared to MIRDcell and they lack user-friendly graphic user interfaces.

It should be pointed out that except for the added new features in MIRDcell V3, the underlined modeling concepts and assumptions are the same as those of MIRDcell V2.1. The cell and the cell nucleus are still modeled as concentric spheres. The effect of the shape of the cell on the calculated absorbed dose is usually small (25), except for certain electron energies that have ranges similar to cellular dimensions (46). Furthermore, unlike PARaDIM (47), V3 also assumes a constant size for all the cells in the population. Like MIRDcell V2.1, the dosimetry and bioeffect modeling in MIRDcell V3 does not account for the stochastic variations in the number of alpha-particle decays, hits, and energy deposited. Furthermore, users should be mindful that photons are ignored in this and earlier versions of MIRDcell; photon contributions to the absorbed dose can become significant for large cluster sizes. MIRDcell V3 does not take 
bystander and abscopal effects to account in the model either (48-50). Also, any dose rate effects and temporal effects such as proliferation are not explicitly accounted for in modeling the biologic response. However, as mentioned in MIRD Pamphlet No. 25 (2), this can be compensated for by using suitable values for the LQ parameters in the Radiobiological Parameters tab.

Similar to MIRDcell V2.1, V3 also uses a variation of the LQ model that accounts for selfand cross-doses when the modeling biologic response of cell clusters to different radiation types. The effect of lesion interactions produced by mixtures of self- and cross-dose on biologic response are ignored, rather their effects are considered independently. New in V3, accommodated by the Complex Radiobiological Parameters tab, is a new target region (cytoplasm) and the capacity to adjust the LQ parameters for each individual radiation type. Again, the effects of each radiation type are treated independently as are the effects from absorbed doses arising from decays in different source regions. This approach can underestimate the effect, particularly at high doses. However, the exact mechanisms behind interactions with mixed-LET radiations are not well understood. Various theoretical formulations for bioeffect modeling of mixed radiations have been proposed by both experimental and theoretical groups over the years (51-53). They include the addition of interaction terms between the various radiation insults that can arise. Although our worked examples show that the present MIRDcell bioeffect models behave satisfactorily, the introduction of interaction terms is under investigation for possible inclusion in MIRDcell algorithms. 


\section{Conclusions}

Given the highly non-uniform cellular exposures received in nuclear medicine, designing treatment plans for therapeutic radiopharmaceuticals is challenging. Therefore, the revisions to this software application were developed to improve visualization and understanding of the impact of radionuclide choice, distribution of activity in and among cells, cell dimensions, intercell distances, cluster size, and radiobiological response parameters on the capacity to kill populations of cells. These parameters can play a substantial role in determining the SF of cells and tumor control probability. Accordingly, MIRDcell is a versatile software tool that can be used for educational purposes and design of radiopharmaceutical therapies.

\section{Disclosure}

MIRDcell V2 is patented under USPTO $\underline{9,623,262}$.

\section{Acknowledgments}

Special thanks to the NJMS Rutgers IT Team who created and support the MIRDcell website and maintain the server. Thanks to Adam Kesner for supporting the distribution of MIRDcell via the MIRDsoft platform. Finally, thanks to Caroline Bolch for designing the MIRDcell icon and logo. This work was supported in part by NIH R01CA198073 and 1R01CA245139. 


\section{References}

1. Sgouros G, Bodei L, McDevitt MR, Nedrow JR. Radiopharmaceutical therapy in cancer: clinical advances and challenges. Nat Rev Drug Discov. 2020;19:589-608.

2. Vaziri B, Wu H, Dhawan AP, Du P, Howell RW. MIRD Pamphlet No. 25: MIRDcell V2.0 software tool for dosimetric analysis of biologic response of multicellular populations. $J$ Nucl Med. 2014;55:1557-1564.

3. Bolch WE, Eckerman KF, Sgouros G, Thomas SR. MIRD pamphlet No. 21: a generalized schema for radiopharmaceutical dosimetry--standardization of nomenclature. J Nucl Med. 2009;50:477-484.

4. Makrigiorgos GM, Adelstein SJ, Kassis Al. Limitations of conventional internal dosimetry at the cellular level. J Nucl Med. 1989;30:1856-1864.

5. Goddu SM, Rao DV, Howell RW. Multicellular dosimetry for micrometastases: dependence of self-dose versus cross-dose to cell nuclei on type and energy of radiation and subcellular distribution of radionuclides. J Nucl Med. 1994;35:521-530.

6. Humm JL, Chin LM. A model of cell inactivation by alpha-particle internal emitters. Radiat Res. 1993;134:143-150.

7. Roeske JC, Stinchcomb TG. Dosimetric framework for therapeutic alpha-particle emitters. J Nucl Med. 1997;38:1923-1928.

8. Akabani G, Zalutsky MR. Microdosimetry of astatine-211 using histological images: application to bone marrow. Radiat Res. 1997;148:599-607.

9. Fisher DR. Alpha-particle emitters in medicine. In: Adelstein SJ, Kassis Al, Burt RW, eds. Dosimetry of Administered Radionuclides. Washington, D.C.: American College of Nuclear Physicians; 1990:194-214.

10. Hobbs RF, Song H, Watchman CJ, et al. A bone marrow toxicity model for ${ }^{223}$ Ra alphaemitter radiopharmaceutical therapy. Phys Med Biol. 2012;57:3207-3222.

11. Kwok CS, Prestwich WV, Wilson BC. Calculation of radiation doses for nonuniformly distributed beta and gamma radionuclides in soft tissue. Med Phys. 1985;12:405-412.

12. Wessels BW, Griffith $\mathrm{MH}$. Miniature thermoluminescent dosimeter absorbed dose measurements in tumor phantom models. J Nucl Med. 1986;27:1308-1314.

13. Humm JL, Cobb LM. Nonuniformity of tumor dose in radioimmunotherapy. J Nucl Med. 1990;31:75-83.

14. Sgouros G, Chiu S, Pentlow KS, et al. Three-dimensional dosimetry for radiotherapy treatment planning. J Nucl Med. 1993;34:1595-1601.

15. Erdi AK, Wessels BW, DeJager R. Tumor activity confirmation and isodose curve display for patients receiving iodine-131-labelled 16.88 human monoclonal antibody. Cancer. 1994;73:932-944.

16. Humm JL, Howell RW, Rao DV. Dosimetry of Auger electron emitting radionuclides: Report No. 3 of the AAPM Nuclear Medicine Task Group No. 6. Med Phys. 1994;21:19011915.

17. Neti PV, Howell RW. Isolating effects of microscopic nonuniform distributions of ${ }^{131}$ I on labeled and unlabeled cells. J Nucl Med. 2004;45:1050-1058. 
18. Howell RW, Bishayee A. Bystander effects caused by nonuniform distributions of DNAincorporated ${ }^{125}$ I. Micron. 2002;33:127-132.

19. Neti PV, Howell RW. When may a nonuniform distribution of ${ }^{131} \mathrm{I}$ be considered uniform? An experimental basis for multicellular dosimetry. J Nucl Med. 2003;44:20192026.

20. Neti PVSV, Howell RW. Biological response to nonuniform distributions of ${ }^{210}$ Po in multicellular clusters. Radiat Res. 2007;168:332-340.

21. Kennel SK, Stabin M, Roeske JC, et al. Radiotoxicity of bismuth-213 bound to membranes of monolayer and spheroid cultures of tumor cells. Radiat Res. 1999;151:244-256.

22. Falzone $\mathrm{N}$, Lee $\mathrm{BQ}$, Able $\mathrm{S}$, et al. Targeting micrometastases: the effect of heterogeneous radionuclide distribution on tumor control probability. J Nucl Med. 2018;

23. Zhu C, Sempkowski M, Holleran T, et al. Alpha-particle radiotherapy: for large solid tumors diffusion trumps targeting. Biomaterials. 2017;130:67-75.

24. Goddu SM, Howell RW, Rao DV. Cellular dosimetry: absorbed fractions for monoenergetic electron and alpha particle sources and S-values for radionuclides uniformly distributed in different cell compartments. J Nucl Med. 1994;35:303-316.

25. Goddu SM, Howell RW, Bouchet LG, Bolch WE, Rao DV. MIRD Cellular S values: selfabsorbed dose per unit cumulated activity for selected radionuclides and monoenergetic electron and alpha particle emitters incorporated into different cell compartments. Reston, VA: Society of Nuclear Medicine; 1997. pp. 183.

26. Rajon D, Bolch WE, Howell RW. Lognormal distribution of cellular uptake of radioactivity: Monte Carlo simulation of irradiation and cell killing in 3-dimensional populations in carbon scaffolds. J Nucl Med. 2011;52:926-933.

27. Akudugu JM, Howell RW. Flow cytometry-assisted Monte Carlo simulation predicts clonogenic survival of cell populations with lognormal distributions of radiopharmaceuticals and anticancer drugs. Int J Radiat Biol. 2012;88:286-293.

28. Howell RW, Neti PV. Modeling multicellular response to nonuniform distributions of radioactivity: differences in cellular response to self-dose and cross-dose. Radiat Res. 2005;163:216-221.

29. Howell RW. Advancements in the use of Auger electrons in science and medicine during the period 2015-2019. Int J Radiat Biol. 2020;1-26.

30. Eckerman KF, Endo A. MIRD: Radionuclide Data and Decay Schemes. 2nd. Reston, VA: Society of Nuclear Medicine; 2008. pp. 671.

31. Cole A. Absorption of $20 \mathrm{eV}$ to $50,000 \mathrm{eV}$ electron beams in air and plastic. Radiat Res. 1969;38:7-33.

32. Howell RW, Rao DV, Sastry KSR. Macroscopic dosimetry for radioimmunotherapy: nonuniform activity distributions in solid tumors. Med Phys. 1989;16:66-74.

33. ICRU. Report 49. Stopping Powers and Ranges for Protons and Alpha Particles. Bethesda, MD: International Commission on Radiation Units and Measurements, Bethesda, MD; 1993.

34. Zaider M, Minerbo GN. Tumour control probability: a formulation applicable to any temporal protocol of dose delivery. Phys Med Biol. 2000;45:279-293. 
35. Hobbs RF, Baechler S, Fu DX, et al. A model of cellular dosimetry for macroscopic tumors in radiopharmaceutical therapy. Med Phys. 2011;38:2892-2903.

36. Schuemann J, McNamara AL, Ramos-Mendez J, et al. TOPAS-nBio: an extension to the TOPAS simulation toolkit for cellular and sub-cellular radiobiology. Radiat Res. 2019;191:125-138.

37. Emfietzoglou D, Kostarelos K, Hadjidoukas P, et al. Subcellular S-factors for low-energy electrons: a comparison of Monte Carlo simulations and continuous-slowing-down calculations. Int J Radiat Biol. 2008;84:1034-1044.

38. Kassis Al, Adelstein SJ, Haydock C, Sastry KS. Thallium-201: an experimental and a theoretical radiobiological approach to dosimetry. J Nucl Med. 1983;24:1164-1175.

39. Howell RW, Rao DV, Haydock C. Dosimetry techniques for therapeutic applications of incorporated radionuclides. In: Adelstein SJ, Kassis Al, Burt RW, eds. Dosimetry of Administered Radionuclides. Washington, D.C.: American College of Nuclear Physicians; 1990:215-256.

40. O'Donoghue JA, Bardies M, Wheldon TE. Relationships between tumor size and curability for uniformly targeted therapy with beta-emitting radionuclides. J Nucl Med. 1995;36:1902-1909.

41. Charlton DE. Radiation effects in spheroids of cells exposed to alpha emitters. Int J Radiat Biol. 2000;76:1555-1564.

42. Howell RW, Rajon D, Bolch WE. Monte Carlo simulation of irradiation and killing in three-dimensional cell populations with lognormal cellular uptake of radioactivity. Int J Radiat Biol. 2012;88:115-122.

43. Marcatili S, Pichard A, Courteau A, et al. Realistic multi-cellular dosimetry for ${ }^{177}$ Lulabelled antibodies: model and application. Phys Med Biol. 2016;61:6935-6952.

44. Cai Z, Kwon YL, Reilly RM. Monte Carlo N-Particle (MCNP) modeling of the cellular dosimetry of ${ }^{64} \mathrm{Cu}$ : comparison with MIRDcell $\mathrm{S}$ values and implications for studies of Its cytotoxic effects. J Nucl Med. 2017;58:339-345.

45. Raghavan R, Howell RW, Zalutsky MR. A model for optimizing delivery of targeted radionuclide therapies into resection cavity margins for the treatment of primary brain cancers. Biomed Phys Eng Express. 2017;3:10.1088/2057-1976/aa1086db1089.

46. Siragusa M, Baiocco G, Fredericia PM, et al. The COOLER code: a novel analytical approach to calculate subcellular energy deposition by internal electron emitters. Radiat Res. 2017;188:204-220.

47. Carter LM, Crawford TM, Sato T, et al. PARaDIM: A PHITS-based Monte Carlo tool for internal dosimetry with tetrahedral mesh computational phantoms. I Nucl Med. 2019;60:1802-1811.

48. Blyth BJ, Sykes PJ. Radiation-induced bystander effects: what are they, and how relevant are they to human radiation exposures? Radiat Res. 2011;176:139-157.

49. Canter BS, Leung CN, Fritton JC, et al. Radium-223-induced bystander effects cause DNA damage and apoptosis in disseminated tumor cells in bone marrow. Mol Cancer Res. 2021;19:1739-1750. 
50. Leung CN, Canter BS, Rajon D, et al. Dose-dependent growth delay of breast cancer xenografts in the bone marrow of mice treated with radium-223: the role of bystander effects and their potential for therapy. J Nucl Med. 2020;61:89-95.

51. Zaider $\mathrm{M}$, Rossi $\mathrm{HH}$. The synergistic effects of different radiations. Radiat Res. 1980;83:732-739.

52. Lam GKY. An isoeffect approach to the study of combined effects of mixed radiations the nonparametric analysis of in vivo data. Radiat Res. 1989;119:424-431.

53. Pfuhl T, Friedrich T, Scholz M. Prediction of cell survival after exposure to mixed radiation fields with the local effect model. Radiat Res. 2020;193:130-142. 


\section{Supplemental Data}

\section{Table of Contents}

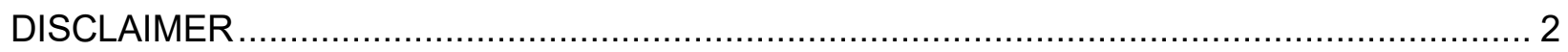

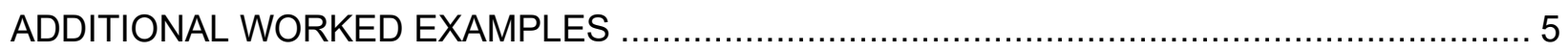

2. Predicting radiotoxicity of ${ }^{111}$ In-EGF distributed in spherical cell clusters ..................... 5

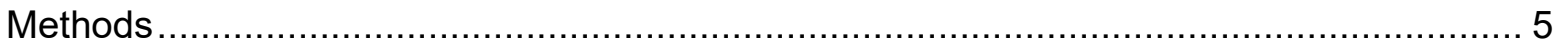

Results and Comparison with Experimental Observations …................................... 9

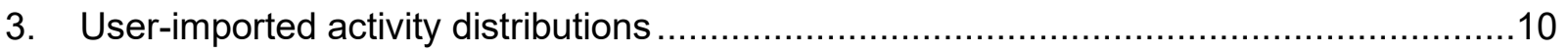

Comparison of MIRDcell and Monte Carlo Simulation ............................................ 14

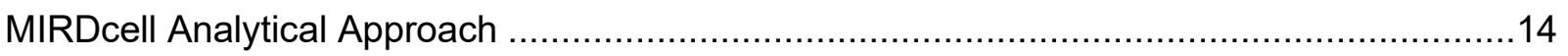

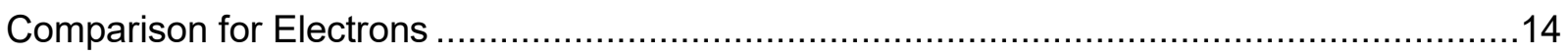

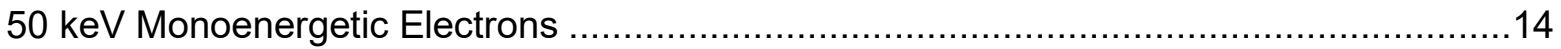

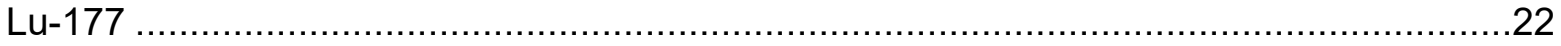

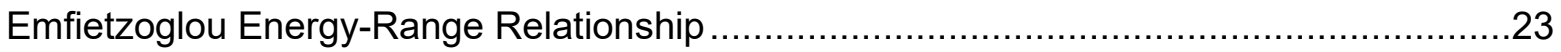

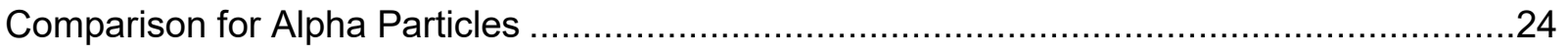

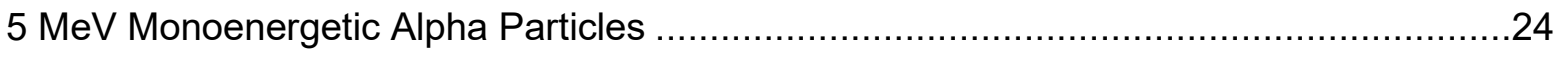

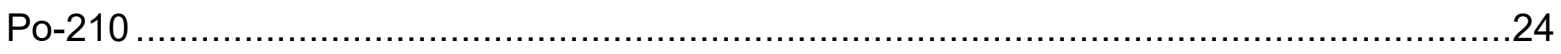

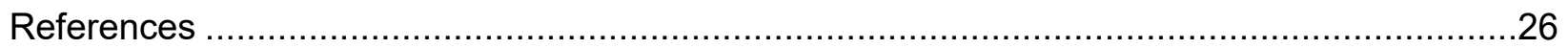

THE JOURNAL OF NUCLEAR MEDICINE • Vol. XX • No. YY • ZZ 2022 Katugampola et al. 


\section{MIRDcell V3・ Katugampola, Wang, Howell}

\section{DISCLAIMER}

1. MIRDcell 3.x is intended for educational and research use only. MIRDcell 3.x has not been approved by the US Food and Drug Administration (FDA) and is not intended for clinical use or use as a medical device. MIRDcell 3.x, all content and links therein, and results generated from the use thereof are not substitutes for medical diagnosis, advice, or treatment of specific medical conditions. A physician should always be consulted for any health problem or medical condition.

2. MIRDcell 3.x was developed by Rutgers, The State University of New Jersey ("RU") with assistance from the Society of Nuclear Medicine and Molecular Imaging (SNMMI)'s Committee on Medical Internal Radiation Dose (MIRD). RU reserves the right to revise, update, or restrict access to MIRDcell 3.x at any time without notice.

3. Your right to access and use MIRDcell 3.x is governed by these Terms of Use. This website (mirdcell.njms.rutgers.edu) contains software necessary for MIRDcell 3.x to function and related copyrighted documentation and materials. You agree not to modify, create derivative works of, decompile, or otherwise attempt to extract source code from MIRDcell 3.x without RU's prior, express, written permission. Aspects of MIRDcell 3.x are protected by U.S. Patent 9,623,262 B2.

4. MIRDcell 3.x is built in the Java environment. Installation of Java SE version 8 is required to use MIRDcell 3.x. You acknowledge and agree that you are solely responsible for obtaining and maintaining such third-party license.

5. You agree to use MIRDcell 3.x for educational and/or research purposes only, and not for any clinical or commercial purposes, including the distribution, licensing or sale of its content to any other person or entity, whether alone or in combination with other materials, or the incorporation of MIRDcell 3.x into any commercial product. Should you display or disclose any results obtained from using MIRDcell 3.x, you agree to indicate that such results were obtained from the use of MIRDcell 3.x.

6. You assume any and all responsibility and liability for your use of MIRDcell 3.x. Any validation of MIRDcell 3.x described herein or elsewhere is not intended to serve as a guarantee of accuracy for any purpose expressed or implied. In no case shall the developers, RU, SNMMI (and its affiliates), or MIRDcell 3.x's distributors or publishers be liable for any damages or consequences, directly or indirectly, arising from any use or misuse of MIRDcell 3.x or results obtained from it.

7. Your IP address may be recorded by Google Analytics or other means for tracking purposes. Usage statistics for MIRDcell 3.x is recorded by RU.

8. You represent, confirm and warrant that you have the full right and authority to provide any data processed within MIRDcell 3.x, to analyze such data, and to obtain results on such data. You further represent, confirm and warrant that the data does not contain any "personally-identifiable information," as such term may be defined under all applicable law.

9. You hereby irrevocably agree to indemnify, defend and hold RU and SNMMI (and its affiliates) harmless from any form of liability from your use of MIRDcell 3.x.

10. IF YOU THINK YOU MAY HAVE A MEDICAL EMERGENCY, PLEASE CALL YOUR DOCTOR OR EMERGENCY SERVICES NUMBER IMMEDIATELY. RU AND SNMMI (AND THEIR AFILIATES) DO NOT RECOMMEND OR ENDORSE ANY TESTS, PRODUCTS, PROCEDURES, OPINIONS, OR OTHER INFORMATION THAT MAY BE MENTIONED THROUGH MIRDCELL 3.x. RELIANCE ON ANY CONTENTS OF MIRDCELL 3.x IS SOLELY AT YOUR OWN RISK.

11. ACCESS TO AND USE OF MIRDCELL 3.X. AND THE CONTENT AND LINKS THEREIN, IS PROVIDED "AS IS", WITHOUT ANY REPRESENTATIONS OR WARRANTIES OF ANY KIND, EXPRESS OR IMPLIED, INCLUDING BUT NOT LIMITED TO THE WARRANTIES OF MERCHANTABILITY, FITNESS FOR A PARTICULAR PURPOSE AND NONINFRINGEMENT. YOU AGREE THAT RU AND SNMMI (AND ITS AFFILIATES) WILL NOT BE LIABLE FOR ANY CLAIMS, DAMAGES, LOSSES OR OTHER LIABILITY, WHETHER IN AN ACTION OF CONTRACT, TORT OR OTHERWISE, ARISING FROM, OUT OF, IN RELIANCE OF OR IN CONNECTION WITH MIRDCELL 3.X.

12. You agree to not use any trademarks or service marks owned or controlled by RU and/or SNMMI (and their affiliates). Nothing contained on this site should be construed as granting, by implication, estoppel, or otherwise, any license or right to use any trademark and/or service mark displayed on this site or any other trademarks or service marks owned or controlled by RU and/or SNMMI (and its affiliates) without the prior written permission of RU or SNMMI, as applicable.

13. To contact us, submit your information to the following: rhowell@rutgers.edu or contact@mirdsoft.org. 
Table 1. Features Supported by Freeware Multicellular Dosimetry Programs

\begin{tabular}{|c|c|c|c|c|}
\hline & $\begin{array}{l}\text { PARADIM } \\
(1)\end{array}$ & $\begin{array}{c}\text { COOLER } \\
(2)\end{array}$ & $\begin{array}{l}\text { MIRDcell } \\
\text { V2.1 }\end{array}$ & $\begin{array}{l}\text { MIRDcell } \\
\text { V3* }^{*}\end{array}$ \\
\hline \multicolumn{5}{|l|}{ Radionuclides } \\
\hline monoenergetic alpha & & & + & + \\
\hline monoenergetic electron & & & + & + \\
\hline user defined & & & + & + \\
\hline MIRD decay schemes $(>200)$ & & & + & + \\
\hline $\begin{array}{l}\text { MIRD decay schemes with beta } \\
\text { energy spectra }(>200)\end{array}$ & & & + & + \\
\hline PHITS radionuclide library & + & & & \\
\hline small selection of radionuclides & & + & & \\
\hline \multicolumn{5}{|l|}{ Cell Geometry } \\
\hline sphere & & & + & + \\
\hline \multicolumn{5}{|l|}{ ellipsoid } \\
\hline \multicolumn{5}{|l|}{ cube } \\
\hline user defined & + & + & & \\
\hline distribution of cell sizes & + & & & \\
\hline$>1$ cell population & & & 2 & 2 \\
\hline \multicolumn{5}{|l|}{ Cell Source Regions } \\
\hline cell $(\mathrm{C})$ & + & + & + & + \\
\hline nucleus $(\mathrm{N})$ & + & + & + & + \\
\hline cytoplasm (Cy) & + & + & + & + \\
\hline cell surface $(\mathrm{CS})$ & & + & + & + \\
\hline$N+C y+C S$ & & & & + \\
\hline \multicolumn{5}{|l|}{ Cell Target Regions } \\
\hline cell & + & & + & + \\
\hline nucleus & + & & + & + \\
\hline cytoplasm & + & & & + \\
\hline cytoplasm + nucleus & & & & + \\
\hline \multicolumn{5}{|l|}{ Bioeffect Modeling } \\
\hline self dose & & & + & + \\
\hline cross dose & & & + & + \\
\hline radiation type & & & & + \\
\hline \multicolumn{5}{|l|}{ dose rate } \\
\hline tumor control probability (TCP) & & & & + \\
\hline
\end{tabular}




\begin{tabular}{|c|c|c|c|c|}
\hline Multicellular Geometry & & & & \\
\hline single cell & + & + & + & + \\
\hline cell pair & & & + & + \\
\hline 2D monolayer (colony) & & & + & + \\
\hline 3D sphere & & & + & + \\
\hline $3 \mathrm{D}$ rod & & & + & + \\
\hline 3D cone & & & + & + \\
\hline 3D ellipsoid & & & + & + \\
\hline control distance between cells & & & + & + \\
\hline control packing density & & & + & + \\
\hline \multicolumn{5}{|l|}{ Activity Distribution } \\
\hline normal & & & + & + \\
\hline lognormal & & & + & + \\
\hline uniform & & & + & + \\
\hline linear (radial) & & & & + \\
\hline exponential (radial) & & & & + \\
\hline polynomial (radial) & & & & + \\
\hline 4-parameter lognormal (radial) & & & & + \\
\hline import user data (radial for sphere) & & & & + \\
\hline cold region for sphere (option) & & & & + \\
\hline cold region for rod (option) & & & & + \\
\hline cold region for cone (option) & & & & + \\
\hline \multicolumn{5}{|l|}{ cold region for ellipsoid (option) } \\
\hline define \%cells labeled & & & + & + \\
\hline \multicolumn{5}{|l|}{ Graphical Output } \\
\hline surviving fraction versus & & & + & + \\
\hline activity per cell & & & + & + \\
\hline decays per cell & & & & + \\
\hline absorbed dose & & & + & + \\
\hline $\begin{array}{l}\text { activity/decays/self-dose/cross- } \\
\text { dose versus }\end{array}$ & & & + & + \\
\hline radial position (sphere) & & & + & + \\
\hline $\begin{array}{l}\text { radial position in axial slices (rod, } \\
\text { cone) }\end{array}$ & & & & + \\
\hline $\begin{array}{l}\text { spatial location of labeled } \\
\text { (live/dead) and unlabeled } \\
\text { (live/dead) cells }\end{array}$ & & & & + \\
\hline axial slices (sphere, rod, cone) & & & & + \\
\hline 3D live/dead display & & & + & + \\
\hline
\end{tabular}

${ }^{*} \mathrm{~A}$ bold plus sign (+) denotes new feature available in MIRDcell V3 compared to V2.1. 


\section{ADDITIONAL WORKED EXAMPLES}

\section{Predicting radiotoxicity of ${ }^{111}$ In-EGF distributed in spherical cell clusters}

Falzone et al. (3) studied the effect of ${ }^{111} \mathrm{In}$-DTPA-human epidermal growth factor $\left({ }^{111} \mathrm{In}\right.$ EGF) on the MDA-MB-468 human breast cancer cell line grown as spheroids. The response of MDA-MB-468 was measured after a $1 \mathrm{~h}$ or $24 \mathrm{~h}$ treatment with the radiopharmaceutical. In this example, the observed biological responses of the MDA-MB-468 spheroids to ${ }^{111} \mathrm{In}$-EGF is modeled with MIRDcell. This example illustrates how the spatial distribution of the radionuclide within the spheroid, which differs after $1 \mathrm{~h}$ and $24 \mathrm{~h}$ treatments, affects the growth of the spheroids. Using the information given in (3), the 3-D spheroid diameter is estimated to be $\sim 450 \mu \mathrm{m}$ (Fig. $2 \mathrm{~A}$ in Ref. (3)). Falzone et al. reports the cell and nucleus radii of MDA-MB-468 to be $9.46 \mu \mathrm{m}$ and $6.65 \mu \mathrm{m}$ respectively. MIRDcell requires integer values for the radii of the cell and cell nucleus, so $9 \mu \mathrm{m}$ and $7 \mu \mathrm{m}$, respectively, are used for modeling. Based on Fig. 3A in Ref. (3) and the spheroid diameter, ${ }^{111}$ In-EGF is estimated to have penetrated $\sim 20 \mu \mathrm{m}$ into the spheroid after a $1 \mathrm{~h}$ treatment with ${ }^{111} \mathrm{In}-\mathrm{EGF}$. The biologic response of the spheroids is modeled with MIRDcell in the following way.

\section{Methods}

a. From the Source Radiation tab, the $-\beta$ Average Energy Spectrum is selected and from the list, $\mathrm{In}-111$ is selected as the radionuclide. The radiation data are displayed in the Input Data for Calculation box in Supplemental Figure 1.

Cell Source/Target Radiobiological Parameters Multicellular Geometry Drug Planning Output Information Credits

V3.07
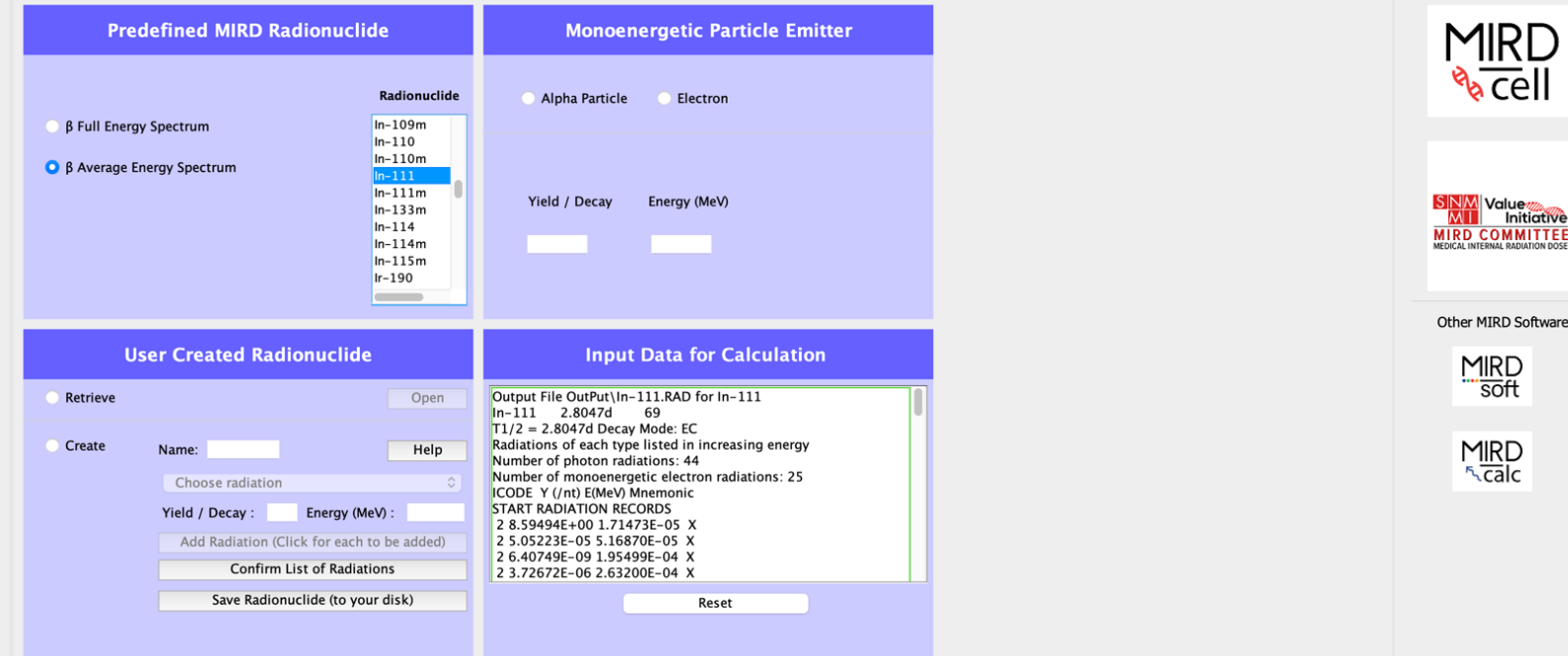

Other MIRD Software

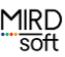

MIRD そălc

Supplemental Figure 1: Radionuclide is selected from "Source Radiation" tab

b. In the Cell Source/Target tab, the radii of the cell and nucleus are set to $9 \mu \mathrm{m}$ and $7 \mu \mathrm{m}$, respectively (Supplemental Fig. 2). The activity is distributed among the cell surface, the cytoplasm and the nucleus according to the data given in Fig. 3D in Ref. (3) for a $1 \mathrm{~h}$ treatment. Supplemental Figure 2 only illustrates the subcellular activity distribution after a $1 \mathrm{~h}$ treatment. The relevant subcellular activity distribution given in Fig. 3D in Ref. (3) after a $24 \mathrm{~h}$ treatment should be used when modeling the corresponding biologic response. 


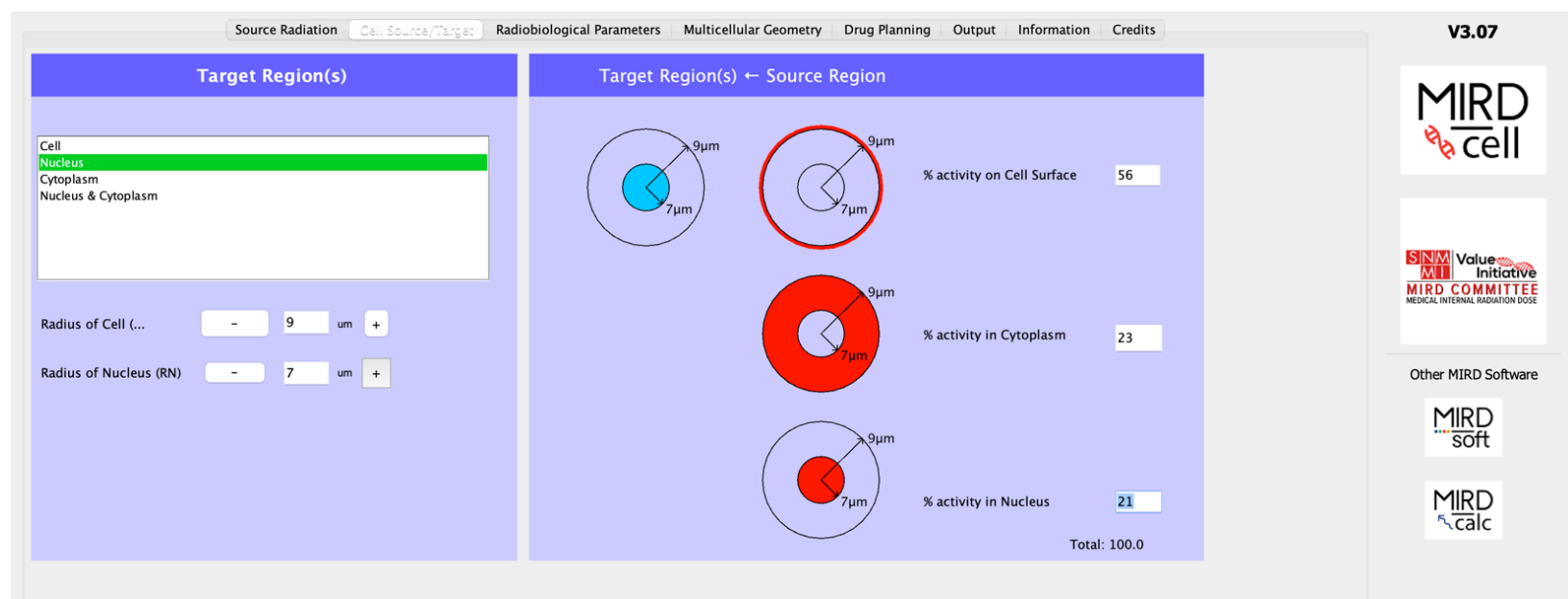

Supplemental Figure 2: Cell and nucleus radii are set. Subcellular activity distribution is set according to experimental observations given in (3). Note that the values given here correspond to the activity distribution after the spheroids are treated with ${ }^{111} \mathrm{In}-\mathrm{EGF}$ for $1 \mathrm{~h}$.

c. Complex Radiobiological Parameters are set in the Radiobiological Parameters tab. In this example, the complex radiobiological parameters, which depend on the radiation type and the source-target regions, are used instead of the simple radiobiological parameters. In the modeling conducted by Falzone et al. (3), the LQ parameters $(\alpha, \beta)$ they obtained for MDAMB-468 cells after ${ }^{137} \mathrm{Cs}$ gamma irradiation were used for electrons. Also, in one of the authors' simulations, they have used a relative biological effectiveness (RBE) equal to 4 for the absorbed dose deposited by ${ }^{111}$ In in the nucleus. Accordingly, for this MIRDcell example, the LQ parameter $\alpha$ is increased by a factor of 4 for Auger electrons for the $\mathrm{N} \leftarrow \mathrm{N}$ target $\leftarrow$ source region, compared to other target $\leftarrow$ source regions (Supplemental Fig. 3 ). It is important to note that, since ${ }^{111}$ In produces some internal conversion electrons (IE) in the decay process, one must pay close attention to the LQ parameters used in the $\beta+, \beta-$, IE row (see Supplemental Fig. 3). In this example, we have used the $\alpha$ values given in (3) for ${ }^{137} \mathrm{Cs}$ gamma irradiation for the $\beta+, \beta$-, IE row. The $\alpha$-particle LQ parameter values are left as default values because these will not affect this simulation. 


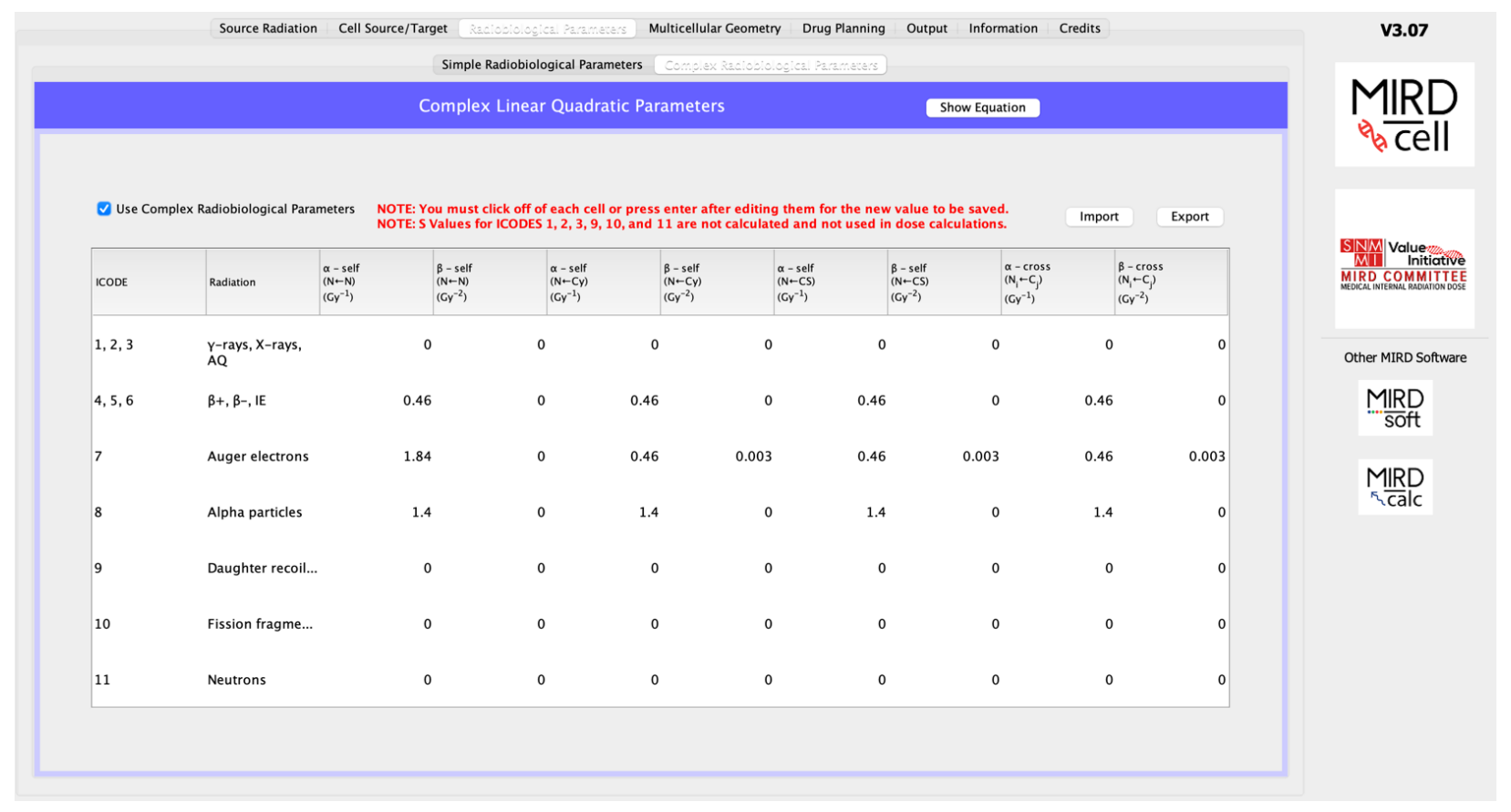

Supplemental Figure 3: Complex radiobiological parameters are set.

d. In the Multicellular Geometry < 3-D Cluster tab, the spheroid radius is set to $225 \mu \mathrm{m}$ and the distance between cells is adjusted until a packing density of 0.17 is achieved (Supplemental Fig. 4). This matched the packing density of 0.17 that was used by Falzone et al. in the Random Close-Packed (RCP) algorithm of their Monte Carlo model (3). This packing density is achieved when the distance between the cells is set to $26 \mu \mathrm{m}$. A drug penetration depth of $20 \mu \mathrm{m}$ is set in accordance with the results given in (3). The product of the Max mean Activity per Cell (All Cells) (Bq) and the Time integrated activity coefficient (hr) should match the mean number of decays per cell when the latter is corrected to seconds. It is advised to start with $100 \%$ as the Percentage of cells that are Labeled (\%) and let the program calculate the maximum number of labeled cells that can be accommodated within the drug penetration depth given other dimensional restrictions. This can be done by clicking the Compute button once. Alternatively, if there is a specific desired percentage, set the value prior to clicking the Compute button. As explained in the previous example, from the Output tab, the results as well as all the parameter values can be saved. 


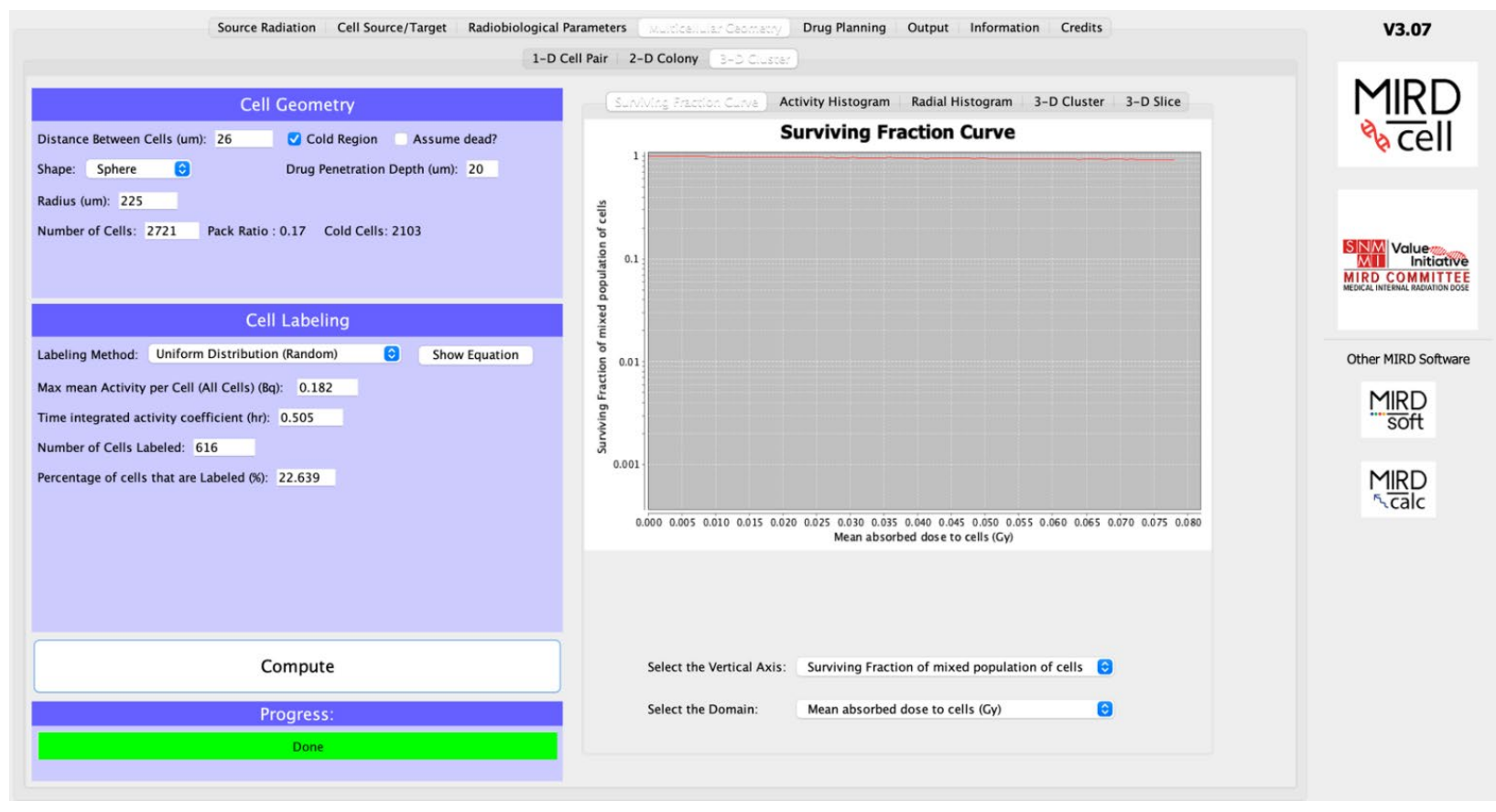

Supplemental Figure 4: Multicellular Geometry tab. The surviving fraction as a function of absorbed dose is plotted on the right. Note that all the parameters and results are for a $1 \mathrm{~h}$ treatment with ${ }^{111} \mathrm{In}$-EGF.

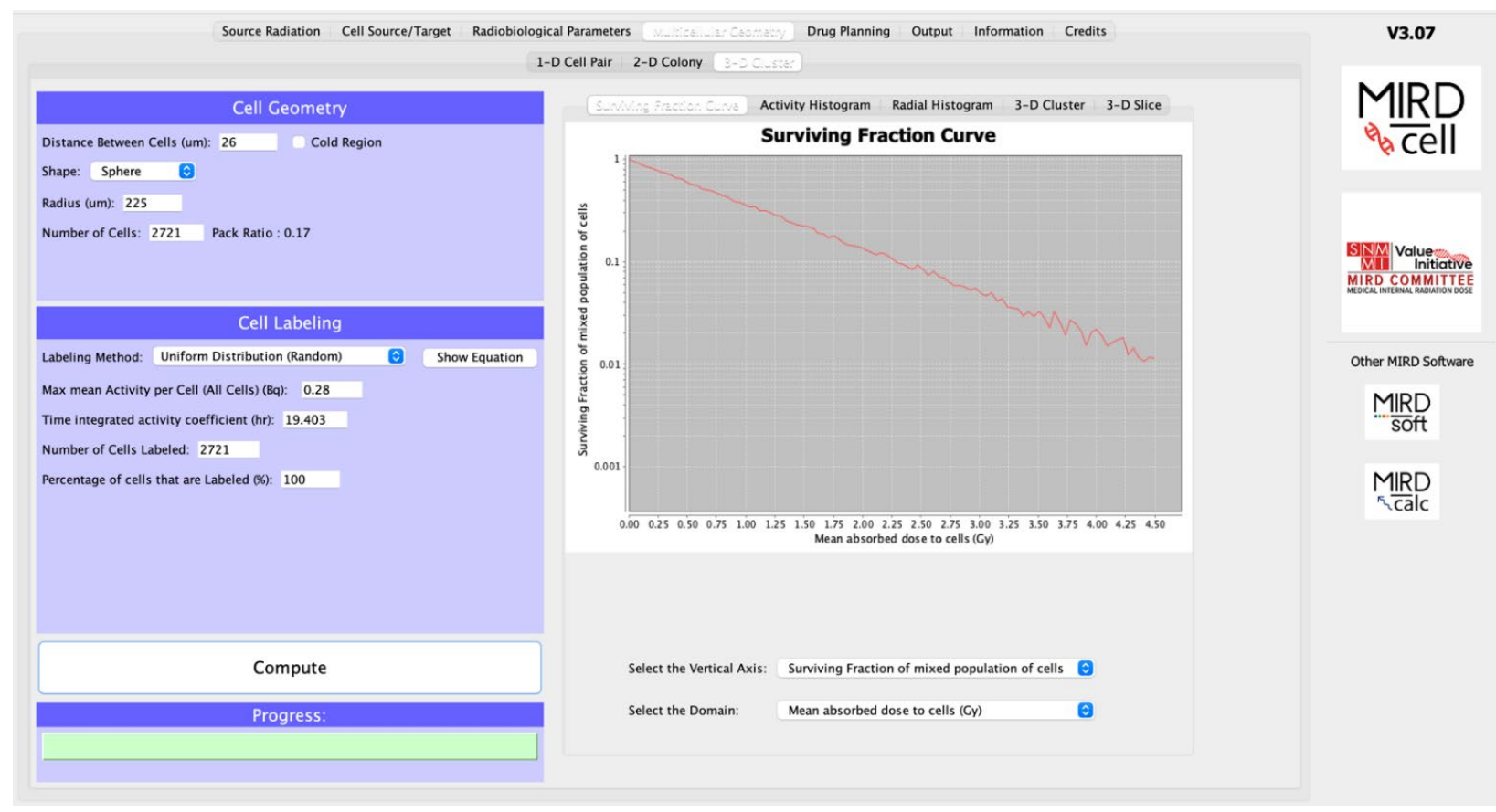

Supplemental Figure 5: Multicellular Geometry tab. Parameter values used in the simulation for a $24 \mathrm{~h}$ treatment with ${ }^{111} \mathrm{In}-\mathrm{EGF}$ are shown in relevant fields. 
e. Similar modeling with MIRDcell can be done for the $24 \mathrm{~h}$ treatment with ${ }^{111} \mathrm{In}-\mathrm{EGF}$. The activity distribution in this case is: $62 \%$ (cell surface), 22\% (cytoplasm), 16\% (nucleus). Other relevant parameter values are shown in Supplemental Figure 5 . Note that, a Drug Penetration Depth (um) is not specified here (i.e. $\square$ Cold Region box is not checked) because the ${ }^{111}$ In-EGF is fully internalized within the spheroid.

\section{Results and Comparison with Experimental Observations}

The data obtained from Falzone et al. (3) showed that after the spheroids were treated with ${ }^{111} \mathrm{In}$-EGF for $1 \mathrm{~h}$, the ${ }^{111} \mathrm{In}$ accumulated mainly on the periphery of the spheroid (Supplemental Fig. 6A). In contrast, after a 24-h treatment, it was fully internalized (Supplemental Fig. 6B). These distributions were recapitulated by MIRDcell in Supplemental Figure 6C and Supplemental Figure 6D which illustrate tomographic sections of MDA-MB-468 spheroids, indicating alive and dead cells, after a $1 \mathrm{~h}$ and $24 \mathrm{~h}$ treatment, respectively.
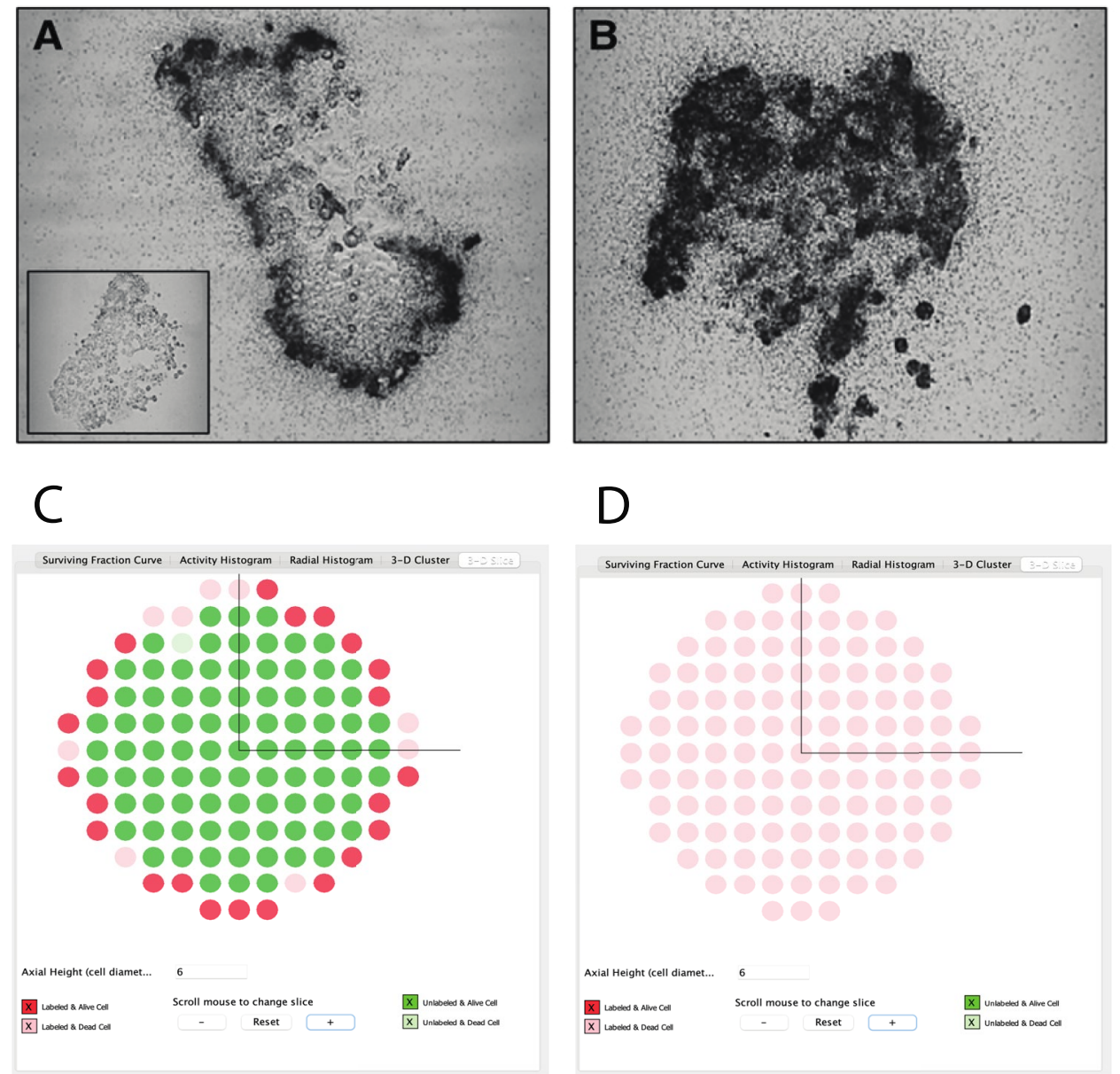

Supplemental Figure 6: Experimental ${ }^{111}$ In-EGF distribution $(A, B)$ in comparison with MIRDcell predictions $(C, D)$ for alive and dead cells in tomographic sections after a $1 \mathrm{~h}(A, C)$ and $24 \mathrm{~h}$ $(B, D)$ treatment with the radiopharmaceutical. $A, B$ were extracted from (3).

Supplemental Figure 7 illustrates a comparison of MIRDcell results for the surviving fraction with experimental and Monte Carlo results reported in (3). The Monte Carlo method used an RBE = 
4 for the absorbed dose from the ${ }^{111}$ In within the nucleus. The MIRDcell predictions are in good agreement with experimental results (Supplemental Fig. 7; numerical values are tabulated in the User Manual).

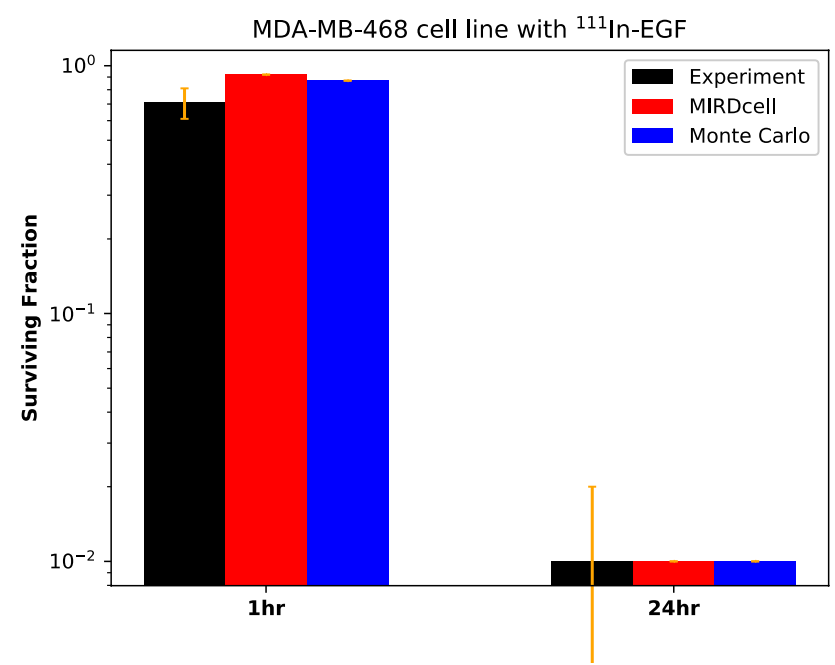

Supplemental Figure 7: Comparison of MIRDcell predictions with experimental and Monte Carlo results for a $1 \mathrm{~h}$ and $24 \mathrm{~h}$ treatment with ${ }^{111} \mathrm{In}-\mathrm{EGF}$.

Because of the relatively short range of the Auger electrons emitted by ${ }^{111} \mathrm{In}$, the total absorbed dose to a labeled cell comes mainly from the self-dose while the cross-dose contribution is insignificant (see Output tab). Therefore, the difference in the surviving fraction following $1 \mathrm{~h}$ and $24 \mathrm{~h}$ treatments is a consequence of the increased percentage of labeled cells in the latter, which in turn increases the total absorbed dose received by the cells in the interior of the cluster.

It is important to note that, when modeling the effects of Auger electron emitters with MIRDcell, the subcellular activity distribution should be considered for multiple reasons. MIRDcell can account for the dependence of the radiotoxicity of Auger emitters based on the source region in which the decays take place.

\section{User-imported activity distributions}

MIRDcell V3 provides the option to import experimentally measured radial activity distributions for spherical clusters. This permits prediction of the biologic response based on the imported distribution rather than the built-in standard distributions (Uniform, Normal, Linear, Exponential, etc). This example illustrates the utility of the user-imported activity distribution feature along with the impact of the activity distribution within the cell cluster on the surviving fraction. 
Supplemental Figure 8: CSV data file.

0,1

$2,1.221402758$

$4,1.491824698$

$6,1.8221188$

$8,2.225540928$

$10,2.718281828$

$12,3.320116923$

$14,4.055199967$

$16,4.953032424$

$18,6.049647464$

$20,7.389056099$

$22,9.025013499$

$24,11.02317638$

$26,13.46373804$

$28,16.44464677$

$30,20.08553692$

$32,24.5325302$

$34,29.96410005$

$36,36.59823444$

$38,44.70118449$

$40,54.59815003$

$42,66.68633104$

$44,81.45086866$

$46,99.48431564$

$48,121.5104175$

$50,148.4131591$

$52,181.2722419$

$54,221.4064162$

$56,270.4264074$

$58,330.2995599$

$60,403.4287935$

$62,492.7490411$

$64,601.8450379$

$66,735.0951892$

$68,897.8472917$

$70,1096.633158$

$72,1339.430764$

$74,1635.98443$

$76,1998.195895$

$78,2440.601978$

$80,2980.957987$

$82,3640.950307$

$84,4447.066748$

$86,5431.659591$

$88,6634.244006$

$90,8103.083928$

$92,9897.129059$

$94,12088.38073$

$96,14764.78157$

$98,18033.74493$

$100,22026.46579$ a. Create a csv file containing the radial activity distribution data. The file must have 2 columns separated by a comma. Column 1 is the radial position and Column 2 is the activity per cell (Supplemental Fig. 8 ). The first radial position of the data should be 0 and the last radial position should match the cluster radius. The data does not have to be absolute activity per cell; relative activity per cell will suffice. With that said, fluorescence intensity data are suitable when fluorescence is used as a surrogate for activity.

b. From the Source Radiation tab, select the desired radionuclide. In this example, select an electron from the Monoenergetic Particle Emitter section. Set the yield to be 1 per decay and the energy to be $1 \mathrm{keV}$. By selecting an electron with such a small energy, it is confined to the cell where it is originated, hence minimizing the cross dose to other cells.

c. From the Cell Source/Target tab, use the default values for the radii of the cell $(5 \mu \mathrm{m})$ and nucleus $(3 \mu \mathrm{m})$. Set nucleus as the "Target region" and assign $100 \%$ activity to the nucleus.

d. Use default values for the LQ parameters in the Radiobiological Parameters < Simple Radiobiological Parameters tab (not shown here).

e. $\quad$ Click the Multicellular Geometry tab. Then select 3-D Cluster. Select Sphere as the shape and set Distance Between Cells (um) to $10 \mu \mathrm{m}$. Set Cluster Radius (um) to $100 \mu \mathrm{m}$.

f. Under Cell Labeling < Labeling Method, choose Import CSV (r, relative A/cell) (Radial) from the drop-down menu. From the window that pops up, navigate to the csv file that contains the data for the activity distribution as a function of radial position and select it. This will import the data to MIRDcell.

g. Set Max mean Activity per Cell (All Cells) (Bq) and Time Integrated activity coefficient (hr) to $0.01 \mathrm{~Bq}$ and $100 \mathrm{~h}$, respectively.

h. Click the Compute button. This will model the biologic response of the cell cluster to the imported activity distribution (Supplemental Fig. 9). 


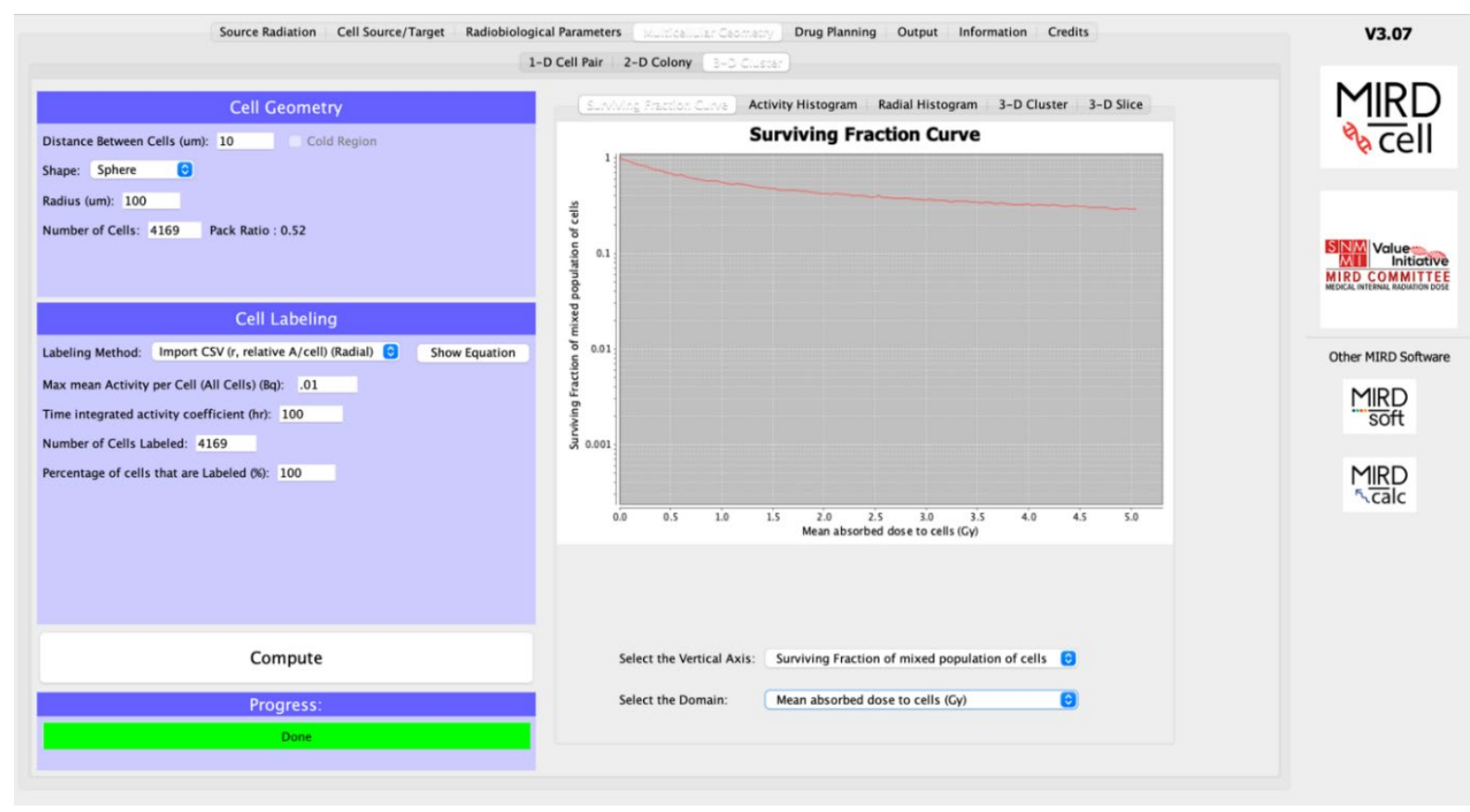

Supplemental Figure 9: Multicellular Geometry tab.

If the same total activity is distributed in a different way within the cluster, the response of the cluster will be different. This can be illustrated in the following way using the same example.

i. Now, without changing anything else, under Labeling Method, select Uniform Distribution (Random) from the drop-down menu. This will change the activity distribution to a uniform distribution instead of the imported distribution. Since everything else in the model is unchanged, the total activity within the cluster remains the same.

j. Click the Compute button.

A comparison of the surviving fraction curves for the two activity distributions is shown in Supplemental Figure 10. Row 1 illustrates the radial variation of the activity of the two distributions (from Radial Histogram tab). Row 2 shows the tomographic sections that correspond to the center of each spheroid (cluster), illustrating the distributions of alive and dead cells for the two activity distributions (from 3-D Slice tab). Row 3 shows the surviving fraction curves, as a function of absorbed dose, for the two activity distributions (from Surviving Fraction Curve tab). The distribution of the activity within the cluster has a major effect on the survival curve. 


\section{MIRDcell V3 • Katugampola, Wang, Howell}

Uniform Activity Distribution

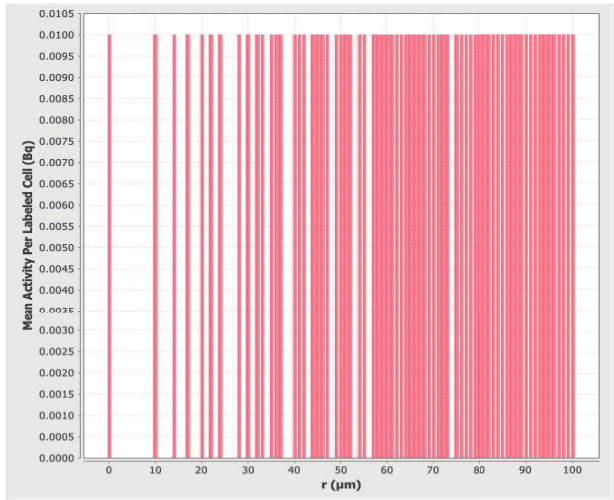

Radial Activity

Distribution

Tomographic

Section

of the Spheroid

showing alive and dead cells

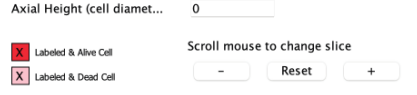

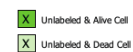

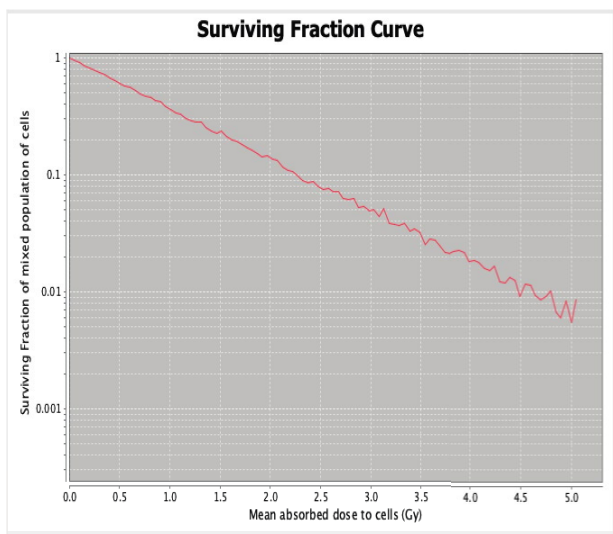

User-uploaded Activity Distribution
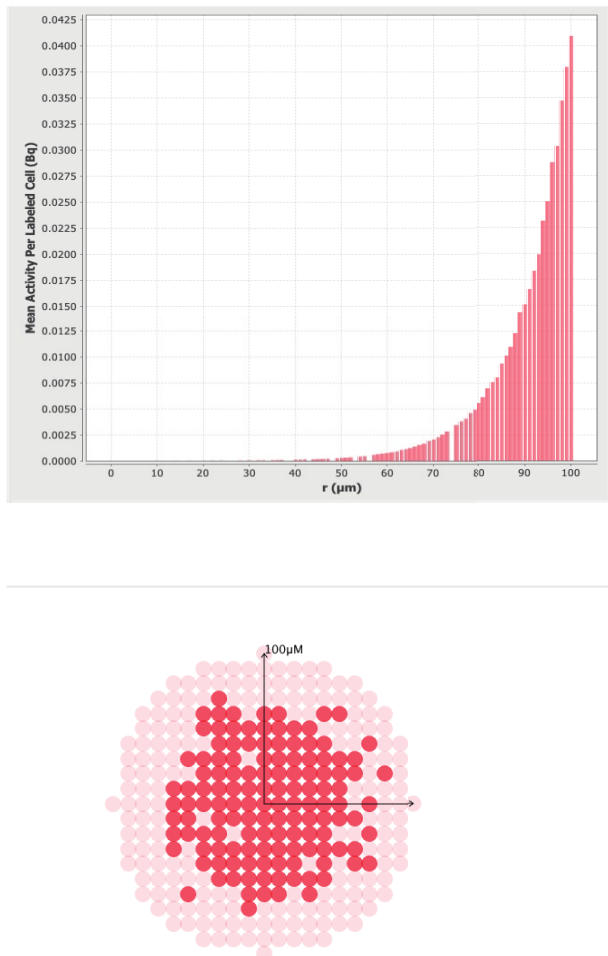

Axial Height (cell diamet... $\quad 0$

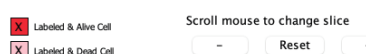

\begin{tabular}{|l|l}
\hline Unlabeled \& Alive Cell \\
\hline Unlabeled \& Dead Cell
\end{tabular}

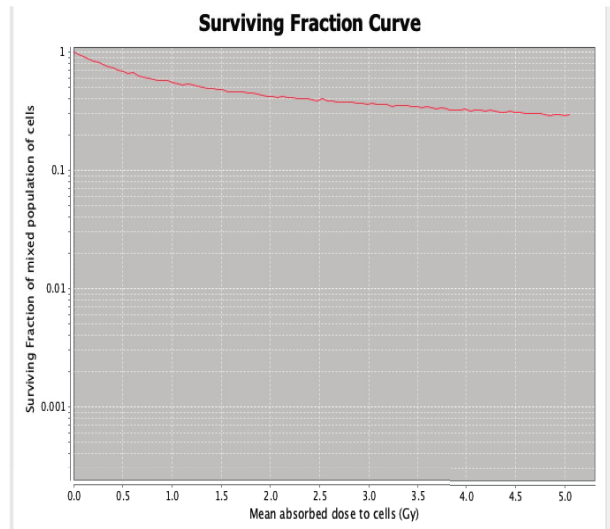

Supplemental Figure 10: The effect of activity distribution on the biologic response. 


\section{Comparison of MIRDcell and Monte Carlo Simulation}

\section{MIRDcell Analytical Approach}

As described by Goddu et al. (4), the energy deposition of electrons and alpha particles is calculated analytically within MIRDcell using the continuously slowing down approximation (CSDA). The fraction of the $i^{\text {th }}$ particle radiation with initial energy, $E_{i}$, emitted from the source region, $r_{S}$, that is absorbed in the target region, $r_{T}$, is called the absorbed fraction $\phi_{i}\left(r_{T} \leftarrow r_{S}\right)$ :

$$
\phi_{i}\left(r_{T} \leftarrow r_{S}\right)=\left.\frac{1}{E_{i}} \int_{0}^{\infty} \psi_{i}\left(r_{T} \leftarrow r_{S}\right) \frac{d E}{d X}\right|_{X\left(E_{i}\right)-x} d x,
$$

where $\psi_{i}\left(r_{T} \leftarrow r_{S}\right)$ is the geometric factor. The geometric factor is the mean probability that a randomly directed vector of length $x$ that starts from a random point within $r_{S}$ and ends within $r_{T}$. The CSDA range of the particle with initial energy, $E_{i}$, is given by $X\left(E_{i}\right)$. The quantity $\left.\frac{d E}{d X}\right|_{X\left(E_{i}\right)-x}$ is the energy loss evaluated at $X\left(E_{i}\right)-x$, the residual range of the particle after passing a distance $x$ through the medium. The $S$ coefficient, $S\left(r_{T} \leftarrow r_{S}\right)$, is the mean absorbed dose to $r_{T}$ per decay in $r_{S}$ :

$$
\mathrm{S}\left(r_{T} \leftarrow r_{S}\right)=\sum_{i=1}^{\mathcal{N}} \frac{\Delta_{i} \phi_{i}\left(r_{T} \leftarrow r_{S}\right)}{m\left(r_{T}\right)}
$$

where $m\left(r_{T}\right)$ is the mass of $r_{T}$ and $\mathcal{N}$ is the number of radiations. The equilibrium dose constant of the $i^{\text {th }}$ radiation, $\Delta_{i}=Y_{i} E_{i}$, where $Y_{i}$ is the radiation yield.

In MIRDcell V3.10 and earlier versions, the cells are assumed to be composed of liquid water and the absorbed fraction for electrons is calculated using Cole's experimental rangeenergy relation for $X$ and, after solving for $E$, its derivative $\left.\frac{d E}{d X}\right|_{X}(5)$. A modification to Cole's expression was used for electrons with ranges below $0.02 \mu \mathrm{m}(6)$. This approach, described in detail in the MIRD Cellular $S$ values monograph along with comparisons of electron $S$ coefficients calculated with the OREC Monte Carlo code (6), was used for electrons at the inception of the algorithms used for MIRDcell because it is valid for electrons with energies ranging from $20 \mathrm{eV}$ to very high energies, and therefore able to accommodate the wide range of electron energies emitted by radionuclides in the form of Auger electrons, internal conversion electrons, and beta particles. In the case of alpha particles, MIRDcell interpolates the ICRU tables for range and stopping power in liquid water to obtain $X$ and $\left.\frac{d E}{d X}\right|_{X}(7)$.

\section{Comparison for Electrons}

\section{$50 \mathrm{keV}$ Monoenergetic Electrons}

S coefficients for cellular self- and cross-dose were calculated with MIRDcell V3.10 and compared with those obtained via a Monte Carlo (MC) simulation performed using TOPAS nBio, a Geant4-based dosimetry tool (8). The simulations were carried out with two cells, each 
composed of liquid water with $10-\mu \mathrm{m}$ cellular radius $\left(R_{C}\right)$ and a $5-\mu \mathrm{m}$ nucleus $\left(R_{N}\right)$ radius. The center-to-center distances between the two cells were varied from $20 \mu \mathrm{m}$ to $60 \mu \mathrm{m}$ at 1- $\mu \mathrm{m}$ intervals and the $\mathrm{S}(\mathrm{N} \leftarrow \mathrm{N})$ were calculated. An identical situation was simulated with TOPASnBio with 8,000,000 source particles distributed uniformly within the nucleus of the source cell. The number of histories used for each source particle was one. In both TOPAS-nBio and MIRDcell, the cell and its nucleus were modeled with two concentric spheres (see Supplemental Fig. 11). The absorbed dose to the target nucleus was scored and the $\mathrm{S}$ coefficients were calculated for each cell separation distance. The "production cuts" for electrons was set at $0.01 \mu \mathrm{m}$ to ensure that each deposition of energy was assigned to the correct subcellular compartment. In TOPAS-nBio (and in Geant4), production cuts control whether a particle is born or not. Therefore, when the primaries no longer have enough energy to produce secondaries which travel at least $0.01 \mu \mathrm{m}$, no more secondaries are produced and the primaries are tracked down to zero energy using continuous energy loss. With these settings, a typical TOPAS-nBio run for a given cell separation, in the Rutgers Amarel super-computing cluster, took about 1.5 hours. Supplemental Figure 12 illustrates the $S$ coefficients $(S(N \leftarrow N)$ and $S(N \leftarrow C y))$ obtained from MIRDcell and TOPAS-nBio simulations for $50 \mathrm{keV}$ electrons. Notable differences can be seen that depend on cell separation distance.

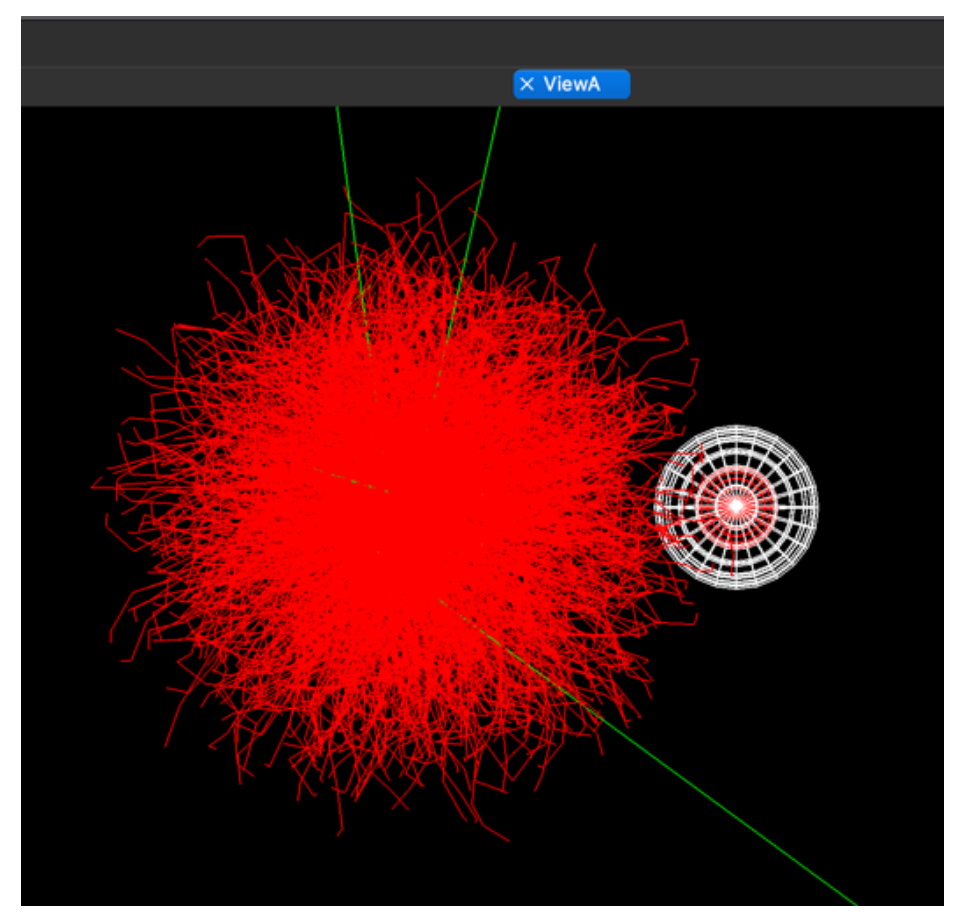

Supplemental Figure 11: Snapshot from the GUI of TOPAS-nBio. The cell separation distance used here is $40 \mu \mathrm{m}$. The target cell and its nucleus are shown on the right in white and red, respectively. The source cell is hidden by the electron tracks (red lines). For clarity, the tracks of only 2000 of the 8,000,000 50-keV electrons are shown here. 

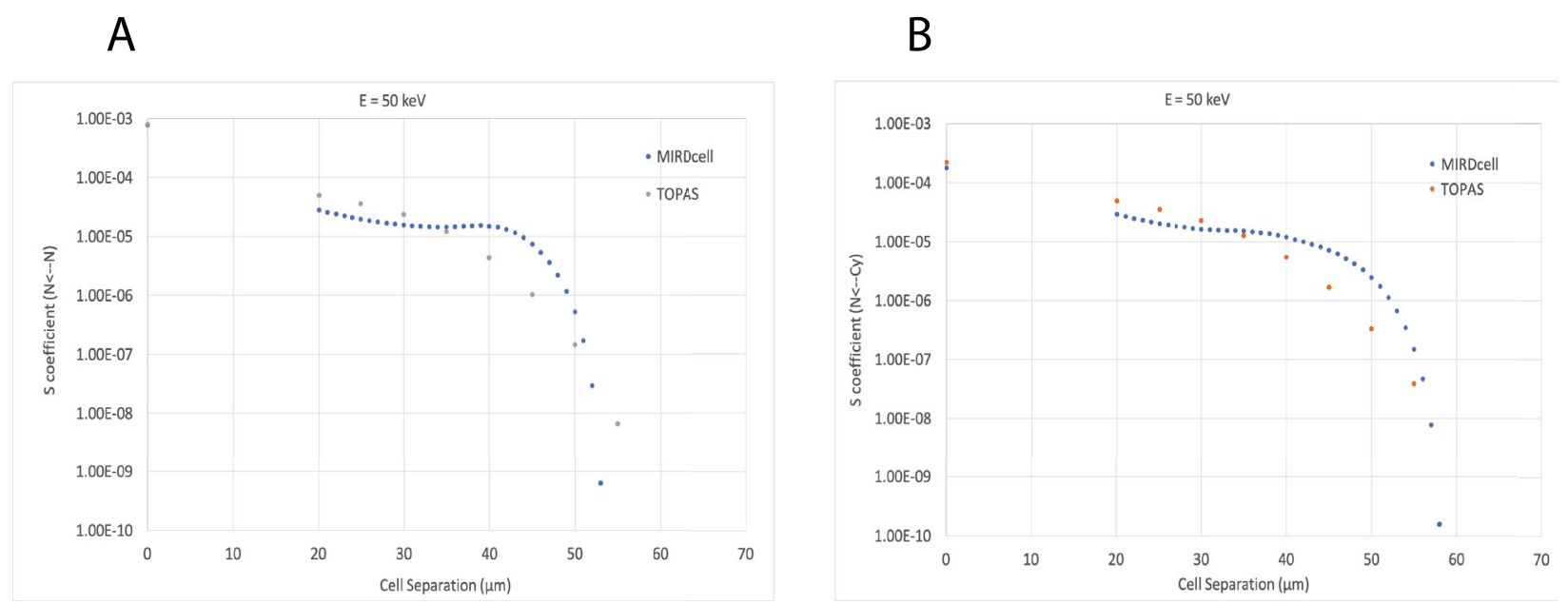

Supplemental Figure 12: $\mathrm{S}$ coefficients from MIRDcell and TOPAS-nBio for $50 \mathrm{keV}$ electron sources distributed uniformly in the nucleus (A) and the cytoplasm (B) of the source cell. The corresponding self $\mathrm{S}$ coefficients in each case are plotted at a cell separation distance of $0 \mu \mathrm{m}$.

While differences in $S$ values are important, the primary purpose of MIRDcell is to predict the surviving fraction and tumor control probability. Accordingly, the effect of these differences on cell survival are analyzed in more detail below. In MIRDcell, the surviving fraction in a 100$\mu \mathrm{m}$ radius spherical cluster of cells was estimated using the default values and the same cell and nucleus dimensions and subcellular activity distributions used above were selected. Additionally, the activity distribution within the cluster was set to be uniform. The parameters used and the resulting surviving fraction curve is shown in Supplemental Figure 13. 


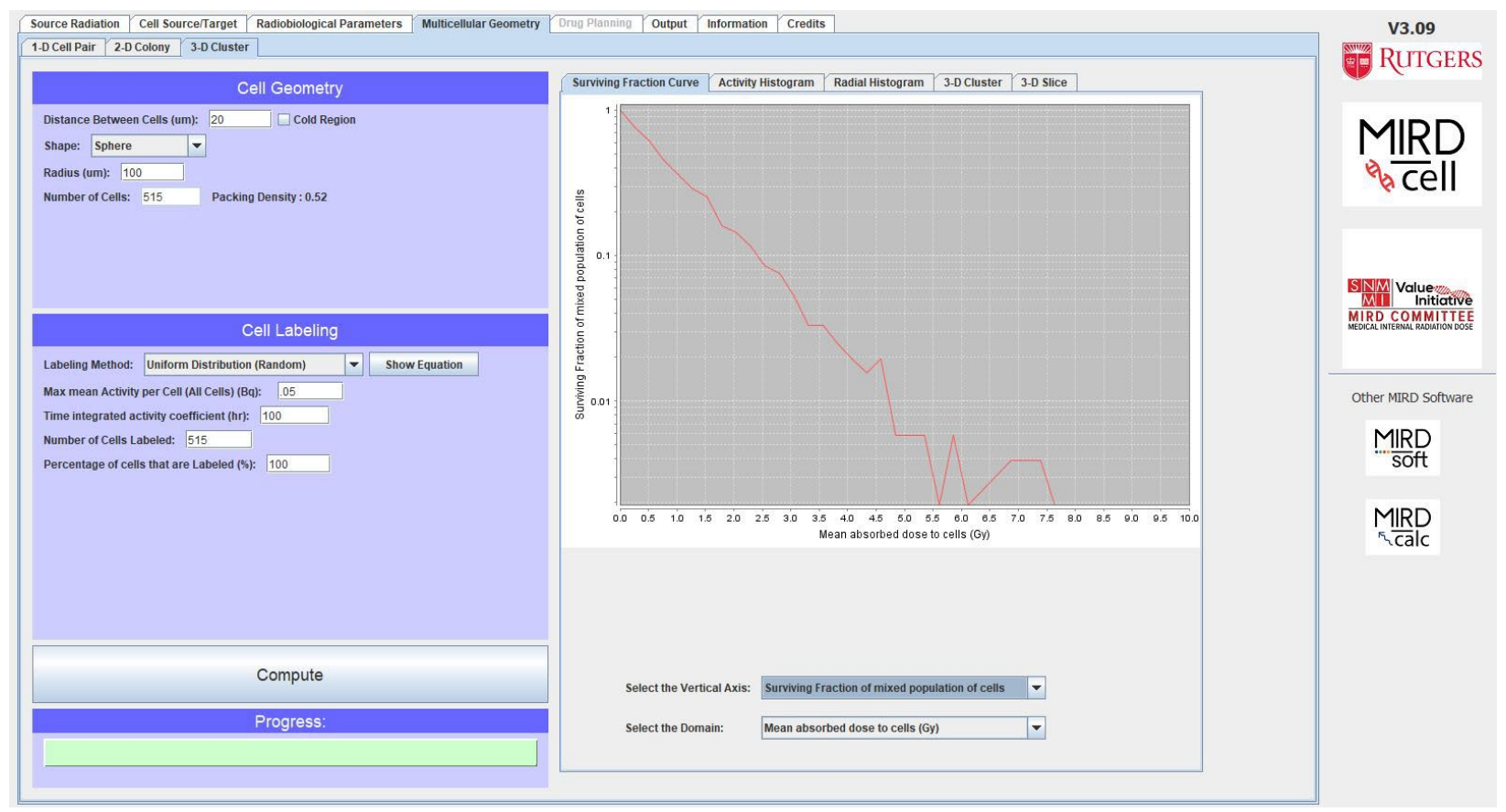

Supplemental Figure 13: Surviving fraction curve resulting from MIRDcell-generated S coefficients.

The $S$ coefficients calculated from TOPAS-nBio were then hard-coded to MIRDcell, replacing the ones that are calculated in a typical MIRDcell simulation, to estimate the surviving fraction under otherwise identical conditions as before. The results are shown in Supplemental Figure 14. The corresponding Tumor Control Probability (TCP) plots are given in Supplemental Figure 15 and Supplemental Figure 16 for MIRDcell-generated and TOPAS-nBio-generated Scoefficients, respectively. It can be seen that, for a uniform activity distribution within the spherical cluster, both the surviving fraction and the TCP plots from MIRDcell-generated Scoefficients are similar to those obtained from TOPAS-nBio-generated S-coefficients. 

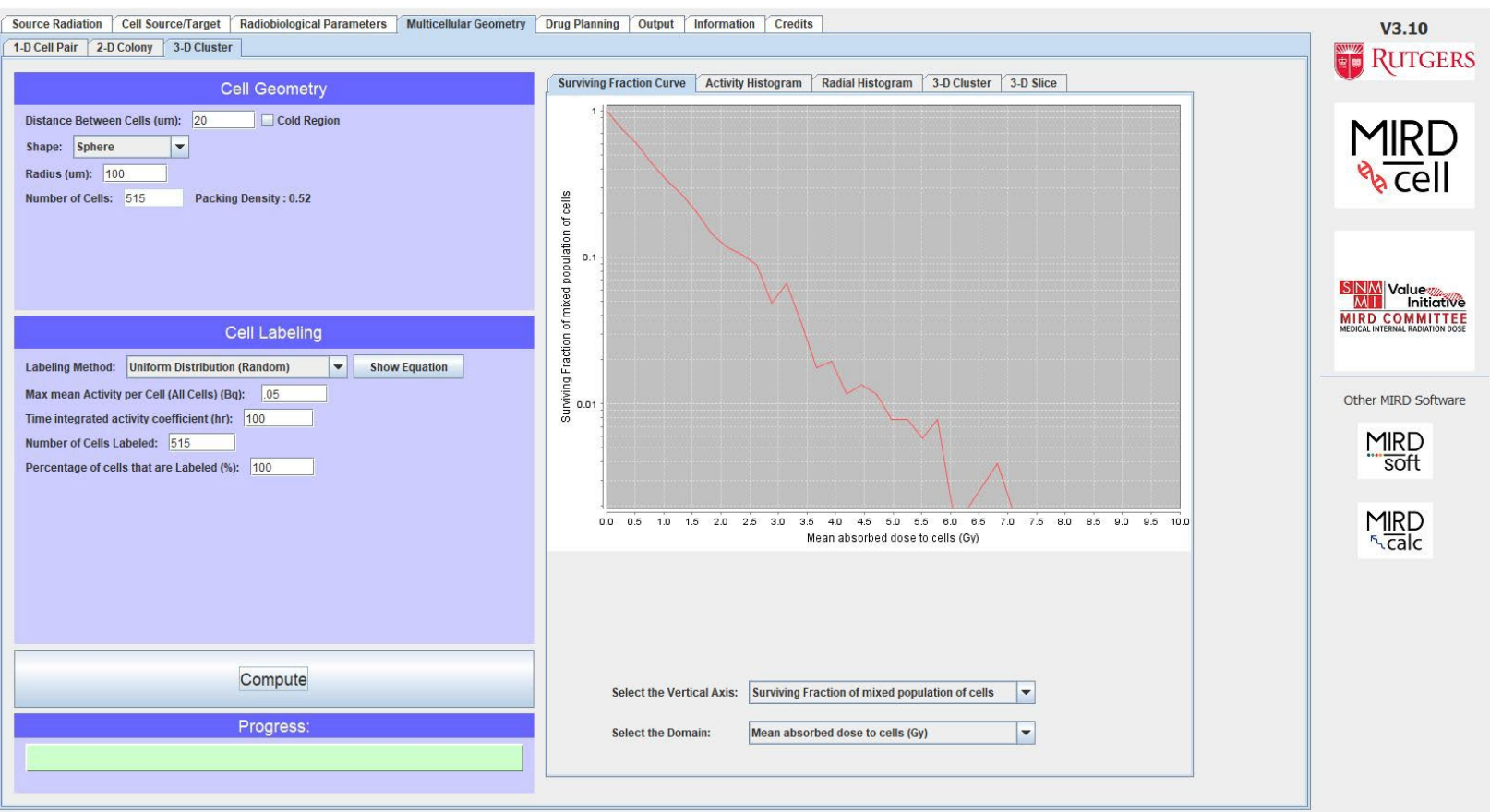

Supplemental Figure 14: Surviving fraction curve resulting from TOPAS-nBio-generated S coefficients.

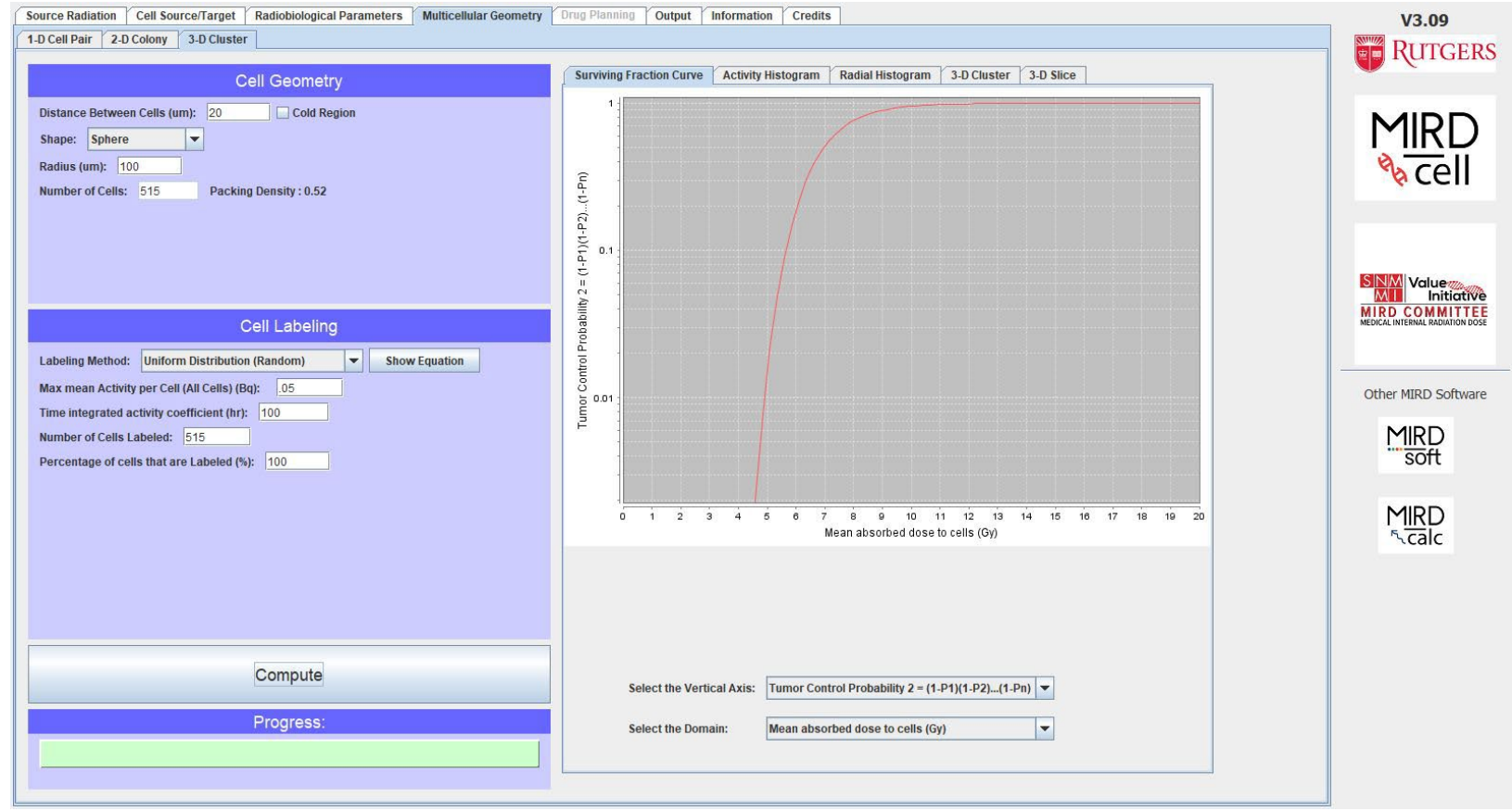

Supplemental Figure 15: Tumor control probability curve resulting from MIRDcell-generated $S$ coefficients. 


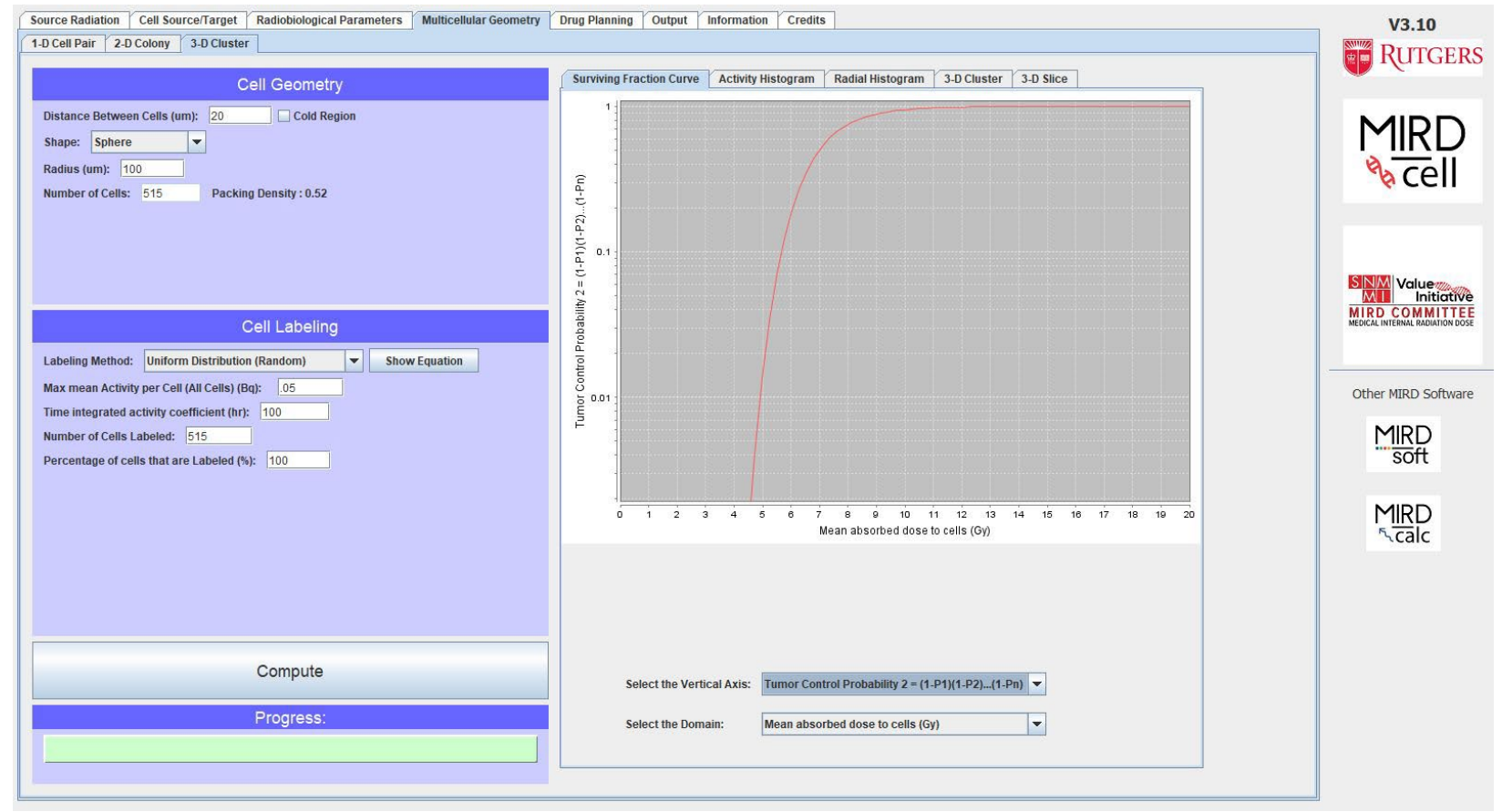

Supplemental Figure 16: Tumor Control Probability curve resulting from TOPAS-nBio-generated $S$ coefficients.

The above exercise was repeated with a radial exponential activity distribution within the spherical cluster, where most of the activity was concentrated in the outer periphery of the cluster. The resulting surviving fraction plots from MIRDcell-generated S-coefficients and TOPAS-nBio-generated S-coefficients are shown in Supplemental Figure 17 and Supplemental Figure 18, respectively. These two survival curves are somewhat different. The SF plot from MIRDcell-generated S-coefficients overestimates the cell kill when compared to the TOPASnBio-generated SF plot. The corresponding TCP plots are modestly different from one another (Supplemental Fig. 19 and Supplemental Fig. 20). These results can be explained by the Cole expression used in MIRDcell, which shows marked peaking of electron $\mathrm{dE} / \mathrm{dR}$ at the end of its track (Supplemental Fig. 12A). When the activity is largely distributed in the outer periphery of the spherical cluster, a significant fraction of electron tracks will end within the cluster towards its center. Therefore, relative to TOPAS-nBio, MIRDcell will enhance the absorbed dose deposited in cells near the center of the cluster. Nevertheless, MIRDcell does a remarkably good job of predicting effect using analytical approaches that only require short computation times. In contrast, the Monte Carlo simulations that are required for multicellular dosimetry require long-computation times which make them presently unsuitable for calculating the multitude of $S$ values needed for the very flexible multicellular modeling scenarios provided by MIRDcell. 


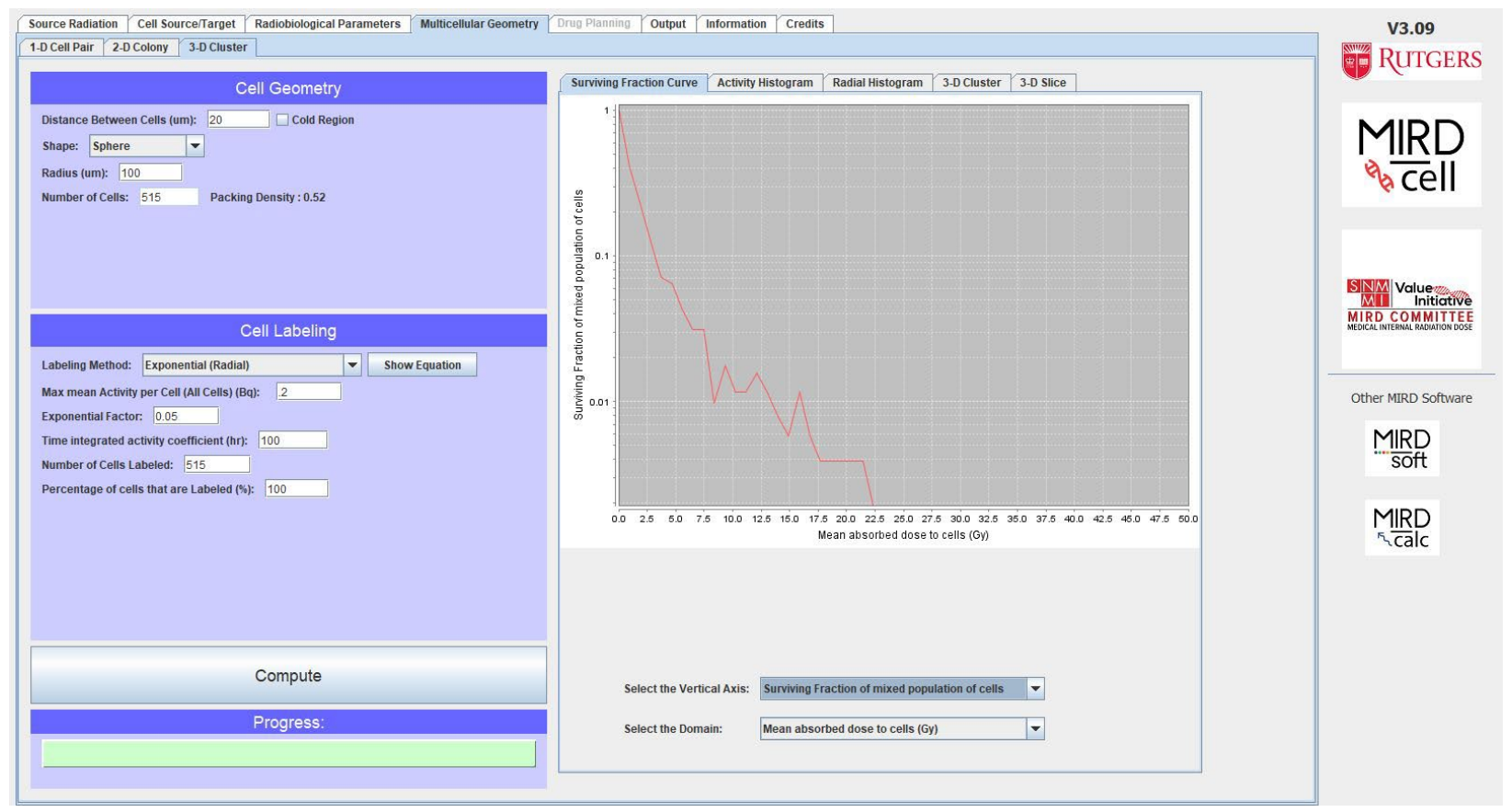

Supplemental Figure 17: Surviving fraction curve resulting from MIRDcell-generated Scoefficients for an exponential activity distribution.

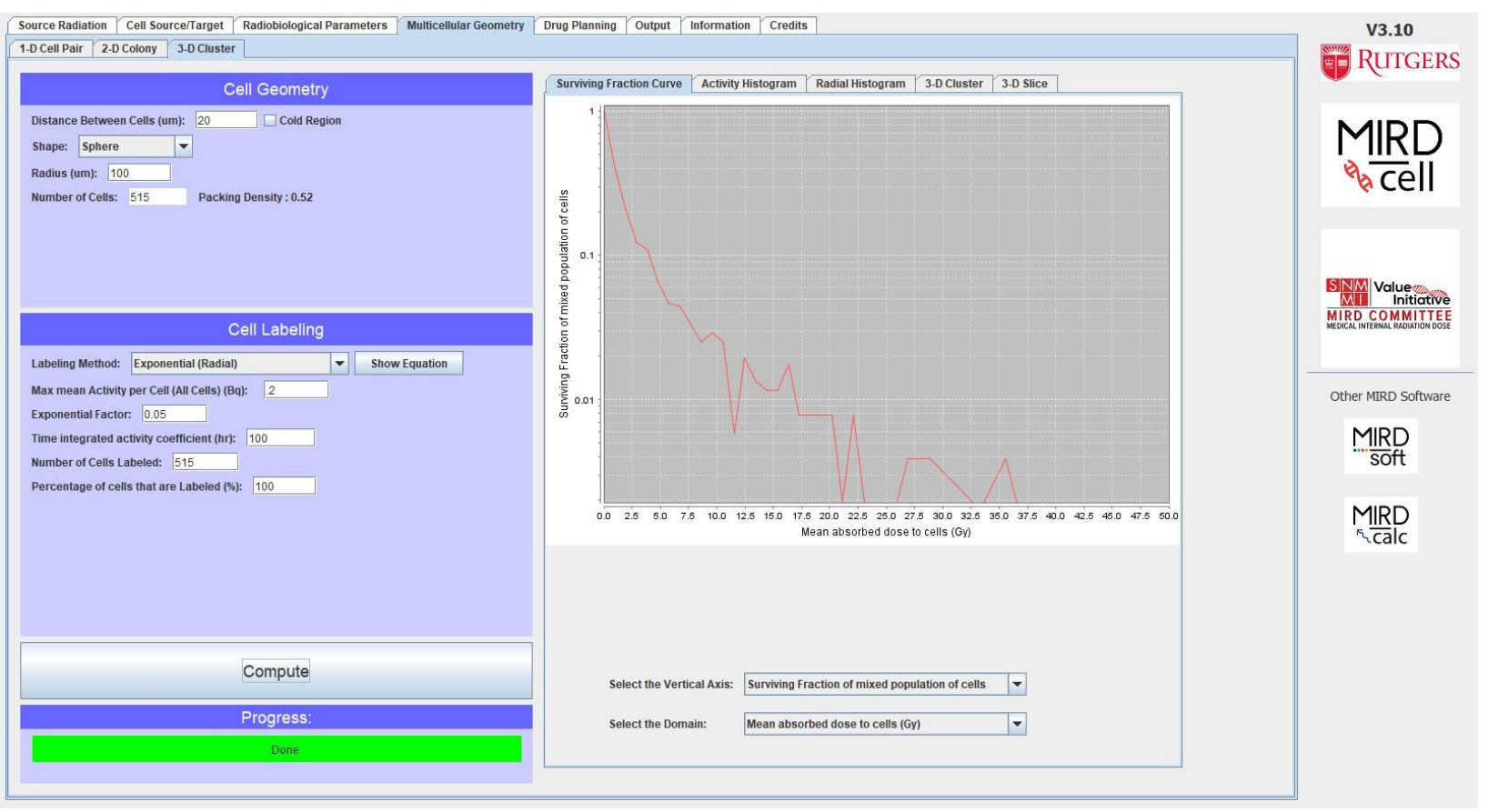

Supplemental Figure 18: Surviving fraction curve resulting from TOPAS-nBio-generated Scoefficients for an exponential activity distribution. 


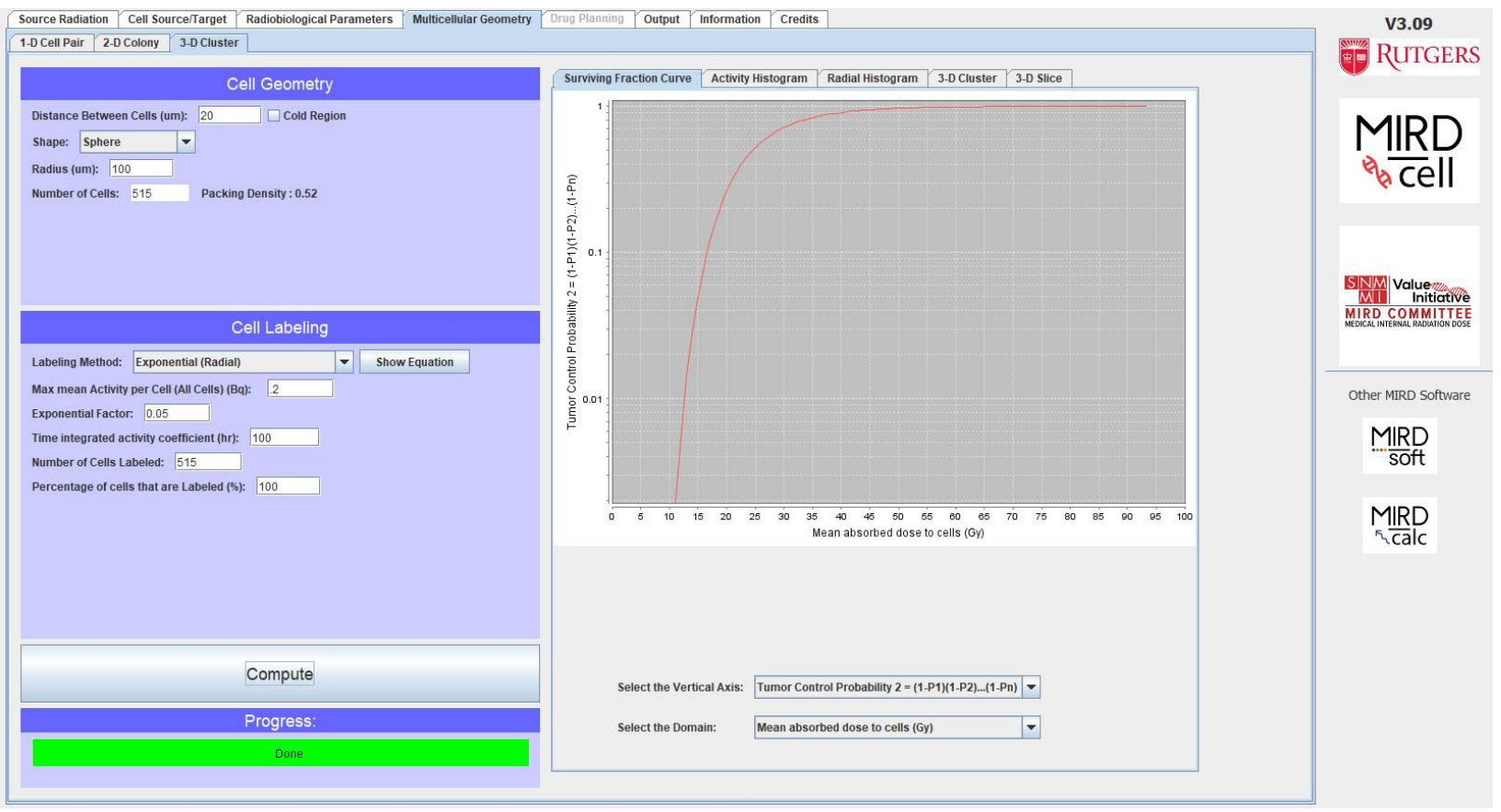

Supplemental Figure 19: Tumor Control Probability curve resulting from MIRDcell-generated S coefficients for an exponential activity distribution.

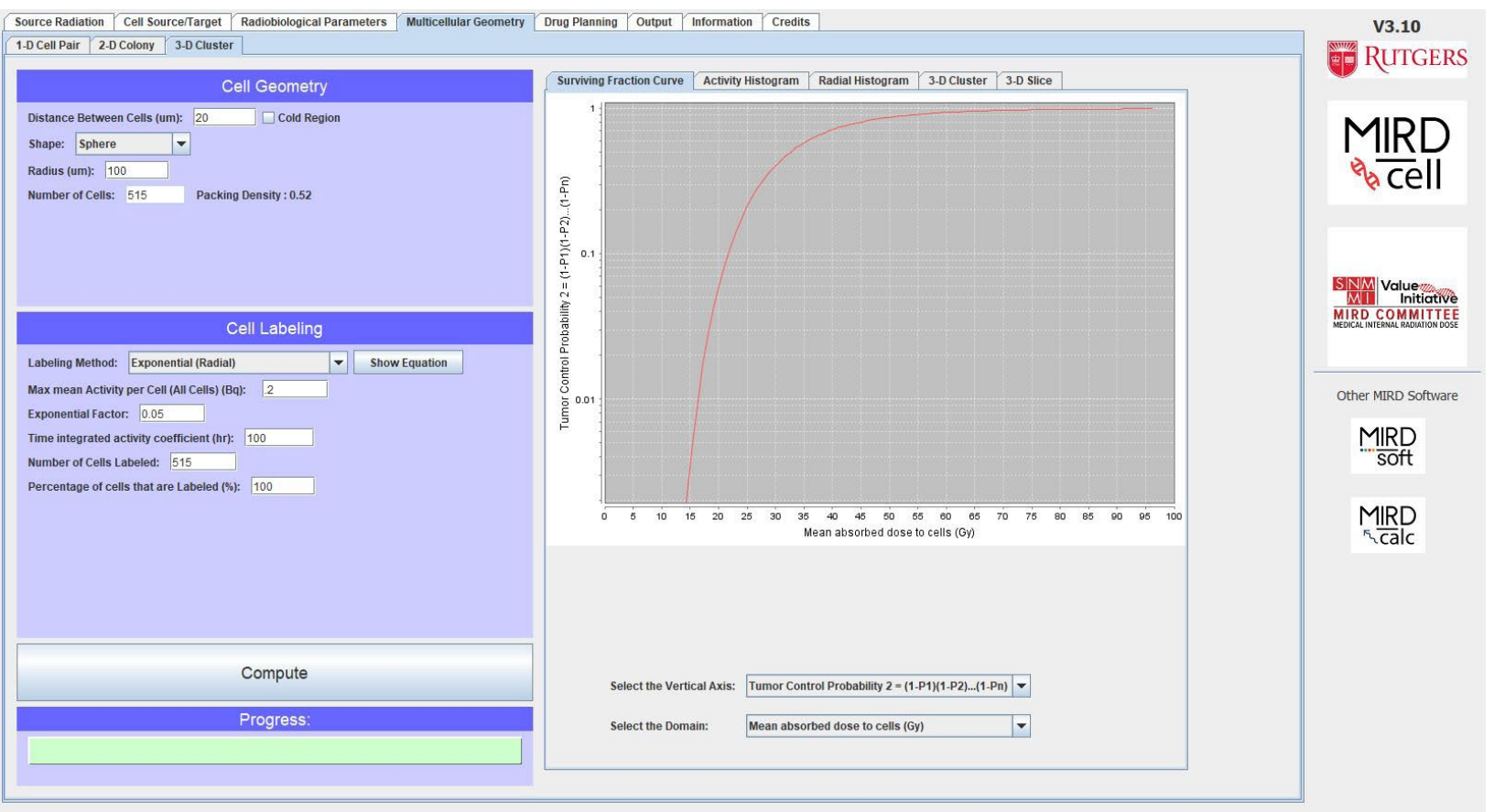

Supplemental Figure 20: Tumor Control Probability curve resulting from TOPAS-nBio-generated $S$ coefficients for an exponential activity distribution. 


\section{Lu-177}

Similar analyses for the $S$ coefficients were done for ${ }^{177} \mathrm{Lu}$ using both MIRDcell and TOPASnBio. In MIRDcell, the full $\beta$ energy spectrum was used to calculate the $S$ coefficients. In TOPAS-nBio, a production cut of $0.01 \mu \mathrm{m}$ was applied for all particles during the simulation. The cellular and nucleus dimensions were kept the same as that in the monoenergetic electronanalysis discussed above. A comparison of the $S$ coefficients calculated with MIRDcell and TOPAS-nBio is shown in Supplemental Figure 21. For the TOPAS-nBio simulation, only a selected set of cell separation distances (MIRDcell $S$ coefficients were calculated at each $\mu \mathrm{m}$ ) were used due to limitations related to excessive computation times.

A

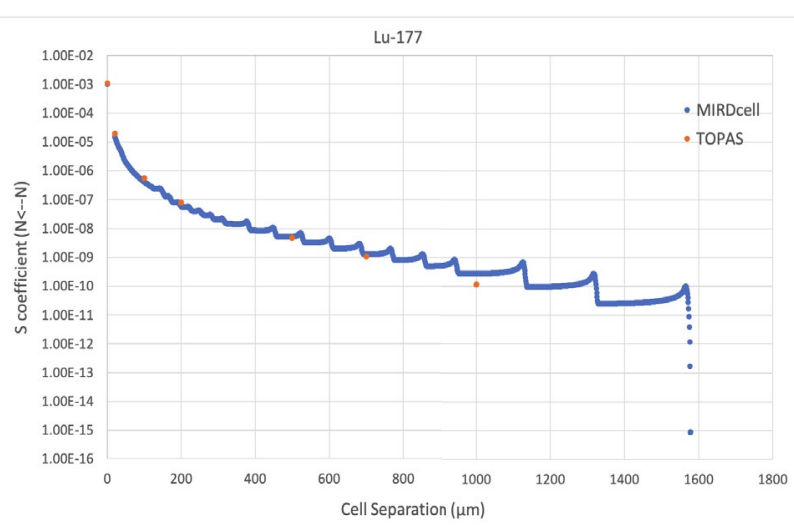

B

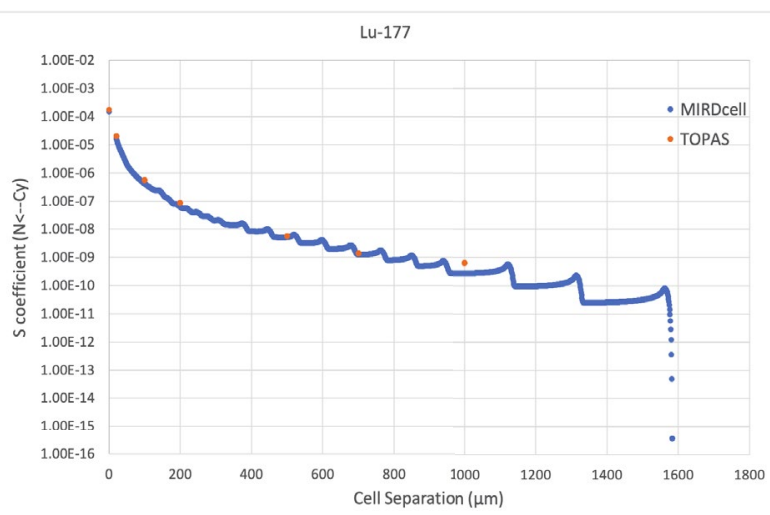

Supplemental Figure 21: S coefficients from MIRDcell and TOPAS-nBio for ${ }^{177} \mathrm{Lu}$ distributed in the nucleus (A) and in the cytoplasm (B) of the source cell. The corresponding self $S$ coefficients in each case are plotted at a cell separation distance of $0 \mu \mathrm{m}$.

The peaks visible in the $S$ coefficients calculated by MIRDcell (also mentioned by Marcatili et al. (9)) are due to two effects. First, MIRDcell uses the Cole's experimental range-energy relation (discussed in the previous section) in calculating the absorbed fraction of the electron energy by the target, which tends to increase towards the track end of an electron (see MIRDcell-calculated S coefficients in Supplemental Fig. 12). Second, the full $\beta$ energy spectrum of ${ }^{177} \mathrm{Lu}$ used in MIRDcell is not a continuous spectrum, instead, it is a discrete spectrum that is logarithmically binned at different electron energies. Therefore, each peak observed for MIRDcell in Supplemental Figure 21 above can be attributed to a track end for a particular electron with a given initial energy. Note that the separation distance between the peaks for each electron increases as the cell separation distance is increased. This is due to the logarithmic binning of the discrete beta spectrum used to approximate the continuous beta spectrum. Smoothing of these peaks can be achieved by increasing the number of energy bins with a consequent increase in computing time. In the limit, a smooth curve could be attained and run times would approach those required for TOPAS-nBio. 


\section{Emfietzoglou Energy-Range Relationship}

Noting the differences between MIRDcell and the TOPAS-nBio results for electrons, we have explored the use of analytical range-energy relationships for electrons other than Cole's. Emfietzoglou et.al (10) have introduced an analytical expression, based on a fit to MCcalculated penetration depth in water data (Fig 3b in Ref. (10)) that is valid for electrons with energies < $10 \mathrm{keV}$. It is given by:

$$
R=a(b+c T)^{d}
$$

where $R$ is the range in $\mathrm{nm}$ and $T$ is the electron energy in keV. The fit parameters a,b,c and $\mathrm{d}$, are: $a=7.511 \times 10^{-6}, b=800.1, c=4702, d=1.783$.

Using MIRDcell, a comparison of $S$ coefficients $(S(C \leftarrow C)$ ) was made between the MIRDcell electron algorithm (Cole's range-energy relation down to residual range of $0.02 \mu \mathrm{m}$ and the Howell expression $(6,11)$ for ranges below that) and an algorithm which uses Cole's relation down to an electron energy of $10 \mathrm{keV}$ and the Emfietzoglou expression given above for lower energies. In the model, $R_{C}=5 \mu \mathrm{m}$ and $R_{N}=3 \mu \mathrm{m}$ were used for both source and target cells, and the source was assigned an electron spectrum with two energies: $50 \mathrm{keV}$ and $100 \mathrm{keV}$, each with a yield of one. A comparison of the calculated $S$ coefficients using the two algorithms is shown in Supplemental Figure 22.

It can be seen from Supplemental Figure 22 that the two expressions show not only similar behavior but also produce comparable results for the $S$ coefficients. The two "bumps" in the curves at around $43 \mu \mathrm{m}$ and $140 \mu \mathrm{m}$ correspond to the CSDA ranges of the $50 \mathrm{keV}$ and the $100 \mathrm{keV}$ electrons respectively. Note that the Cole+Emfietzoglou $\mathrm{S}$ coefficients are larger (up to $20 \%$ ) than the Cole+Howell S coefficients around the $42 \mu \mathrm{m}$ position.

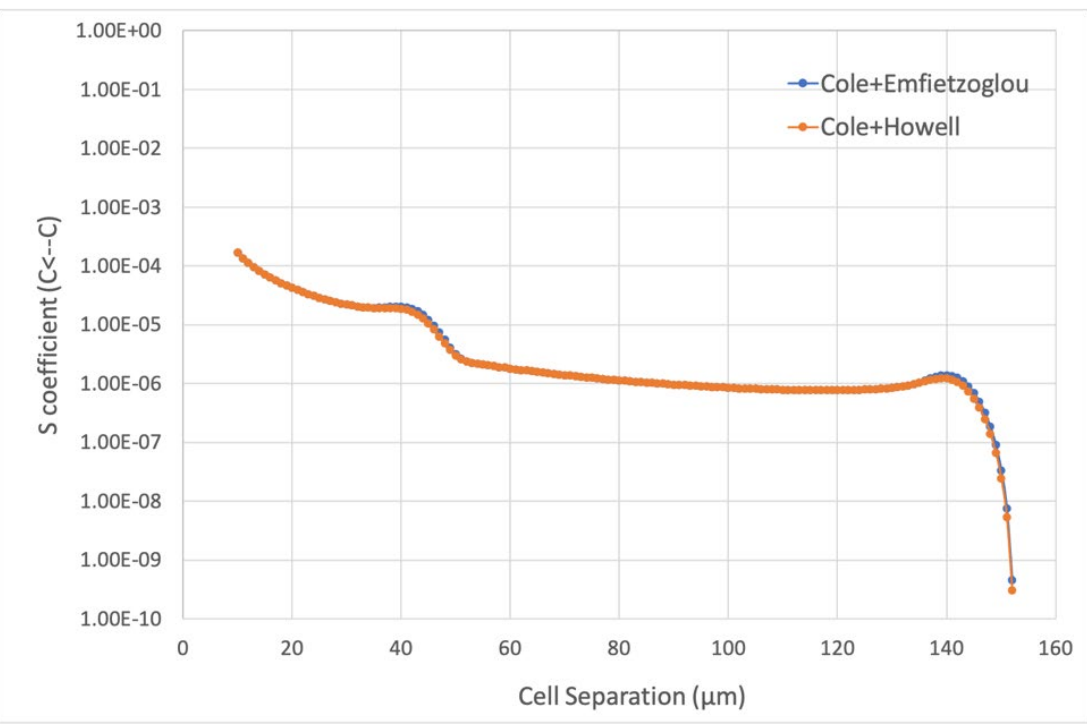




\section{Comparison for Alpha Particles}

\section{MeV Monoenergetic Alpha Particles}

The $S$ coefficients, $S(N \leftarrow N)$ and $S(N \leftarrow C y)$, were calculated for $5 \mathrm{MeV}$ alpha particles using MIRDcell and TOPAS-nBio. The cellular and nucleus dimensions used were $R_{C}=10 \mu \mathrm{m}$ and $R_{N}$ $=5 \mu \mathrm{m}$. In TOPAS-nBio, a production cut of $0.01 \mu \mathrm{m}$ was applied for both alpha particles and electrons during the simulation. A comparison of the MIRDcell-calculated and MC-calculated S coefficients is shown in Supplemental Figure 23. The self S-coefficients are plotted at the cell separation distance of $0 \mu \mathrm{m}$. There is excellent agreement between MIRDcell and TOPAS-nBio.

A

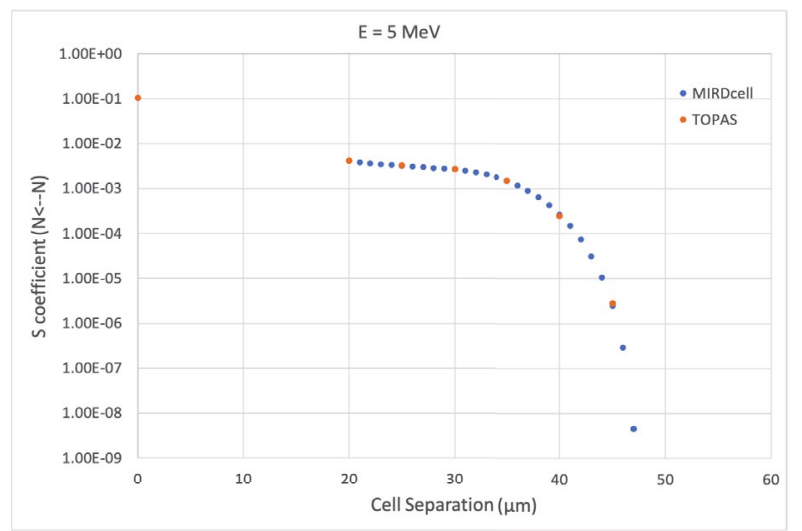

B

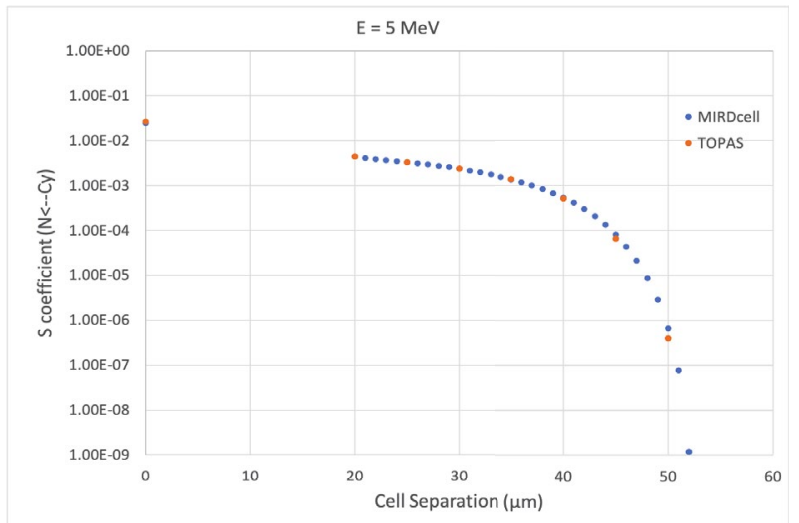

Po-210

A similar analysis was performed for the alpha emitter ${ }^{210} \mathrm{Po}$ using both MIRDcell and TOPAS-nBio. The cell and nucleus dimensions were again kept the same. The results are given in Supplemental Figure 24. The production cut used in the TOPAS-nBio simulations was $0.01 \mu \mathrm{m}$ for both alpha particles and electrons. The tail in the $S$ coefficient curve predicted by MIRDcell for cell separation distances above $52-53 \mu \mathrm{m}$ is due to the conversion electrons in the ${ }^{210} \mathrm{Po}$ spectrum which have a very small yield. The agreement between MIRDcell and TOPAS$\mathrm{nBio}$ is satisfactory for ${ }^{210} \mathrm{Po}$. 
MIRDcell V3 • Katugampola, Wang, Howell

A

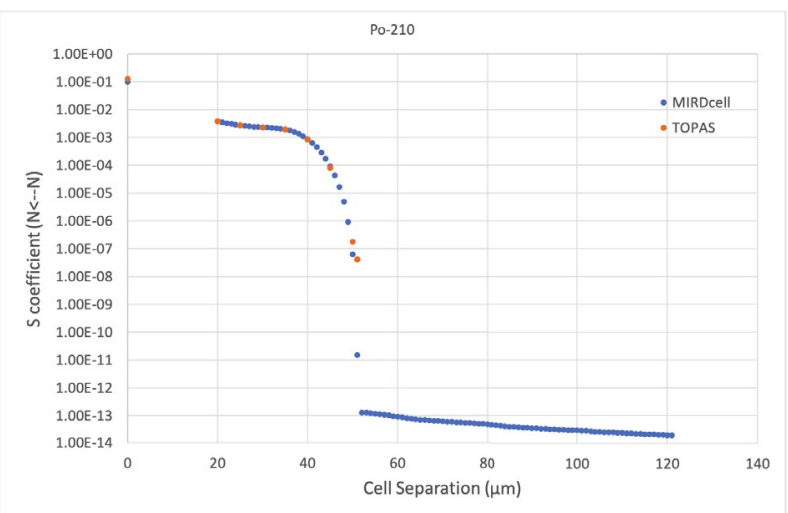

B

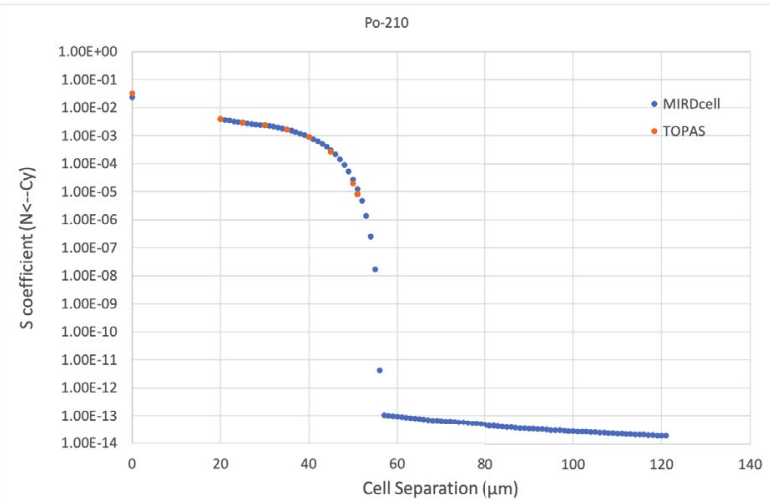




\section{References}

1. Carter LM, Crawford TM, Sato T, et al. PARaDIM: A PHITS-based Monte Carlo tool for internal dosimetry with tetrahedral mesh computational phantoms. I Nucl Med. 2019;60:1802-1811.

2. Siragusa M, Baiocco G, Fredericia PM, et al. The COOLER code: a novel analytical approach to calculate subcellular energy deposition by internal electron emitters. Radiat Res. 2017;188:204-220.

3. Falzone $\mathrm{N}$, Lee $\mathrm{BQ}$, Able $\mathrm{S}$, et al. Targeting micrometastases: the effect of heterogeneous radionuclide distribution on tumor control probability. I Nucl Med. 2018;60:250-258.

4. Goddu SM, Howell RW, Rao DV. Cellular dosimetry: absorbed fractions for monoenergetic electron and alpha particle sources and S-values for radionuclides uniformly distributed in different cell compartments. J Nucl Med. 1994;35:303-316.

5. Cole A. Absorption of $20 \mathrm{eV}$ to $50,000 \mathrm{eV}$ electron beams in air and plastic. Radiat Res. 1969;38:7-33.

6. Goddu SM, Howell RW, Bouchet LG, Bolch WE, Rao DV. MIRD Cellular S values: selfabsorbed dose per unit cumulated activity for selected radionuclides and monoenergetic electron and alpha particle emitters incorporated into different cell compartments. Reston, VA: Society of Nuclear Medicine; 1997. pp. 183.

7. ICRU. Report 49. Stopping Powers and Ranges for Protons and Alpha Particles. Bethesda, MD: International Commission on Radiation Units and Measurements, Bethesda, MD; 1993.

8. Schuemann J, McNamara AL, Ramos-Méndez J, et al. TOPAS-nBio: an extension to the TOPAS simulation toolkit for cellular and sub-cellular radiobiology. Radiat Res. 2019;191:125-138.

9. Marcatili S, Pichard A, Courteau A, et al. Realistic multi-cellular dosimetry for ${ }^{177}$ Lulabelled antibodies: model and application. Phys Med Biol. 2016;61:6935-6952.

10. Emfietzoglou D, Kostarelos K, Hadjidoukas P, et al. Subcellular S-factors for low-energy electrons: a comparison of Monte Carlo simulations and continuous-slowing-down calculations. Int J Radiat Biol. 2008;84:1034-1044.

11. Howell RW, Rao DV, Sastry KSR. Macroscopic dosimetry for radioimmunotherapy: nonuniform activity distributions in solid tumors. Med Phys. 1989;16:66-74. 